\title{
Airfoil noise reductions through leading edge serrations
}

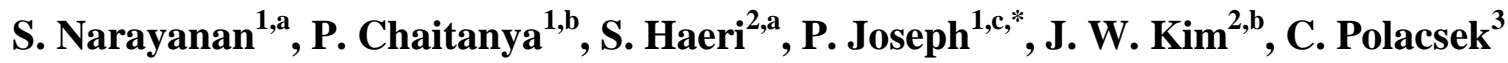 \\ University of Southampton, Highfield, \\ Southampton-SO17 1BJ, UK
}

\begin{abstract}
This paper provides an experimental investigation into the use of leading edge serrations as a means of reducing the broadband noise generated due to the interaction between the aerofoil's leading edge $(L E)$ and impinging turbulence. Experiments are performed on a flat plate in an open jet wind tunnel. Grids are used to generate isotropic homogeneous turbulence. The leading edge serrations are in the form of sinusoidal profiles of wavelengths, $\lambda$, and amplitudes, $2 h$. The frequency and amplitude characteristics are studied in detail in order to understand the effect of $L E$ serrations on noise reduction characteristics and are compared with straight edge baseline flat plates. Noise reductions are found to be insignificant at low frequencies but significant in the mid frequency range $(500 \mathrm{~Hz}$ to $8 \mathrm{kHz})$ for all the cases studied. The flat plate results are also compared to the noise reductions obtained on a serrated NACA-65 aerofoil with the same serration profile. Noise reductions are found to be significantly higher for the flat plates with a maximum noise reduction of around $9 \mathrm{~dB}$ compared with about $7 \mathrm{~dB}$ for the aerofoil. In general, it is observed that the sound power reduction level $(\triangle P W L)$ is sensitive to the amplitude, $2 h$ of the $L E$ serrations but much less sensitive to the serration wavelength, $\lambda$. Thus, this paper sufficiently demonstrates that the $L E$ amplitude act as a key parameter for enhancing the noise reduction levels in flat plates and aerofoils.
\end{abstract}

Keywords: Flat plate, Aerofoil, $L E$ serrations

\footnotetext{
${ }^{1, a}$ Postdoctoral Research Fellow, Acoustics Research Group, Institute of Sound and Vibration Research (ISVR), University of Southampton, Southampton - SO17 1BJ, UK

${ }^{1, \mathrm{~b}} \mathrm{PhD}$ Student, Acoustics Research Group, Institute of Sound and Vibration Research (ISVR), University of Southampton, Southampton SO17 1BJ, UK

2,a Postdoctoral Research Fellow, Aerodynamics and Flight Mechanics Group, University of Southampton, Southampton - SO17 1BJ, UK

${ }^{1, \mathrm{c}}$ Faculty of Engineering and the Environment, Acoustics Research Group, Institute of Sound and Vibration Research (ISVR), University of Southampton, Southampton - SO17 1BJ, UK

${ }^{2, b}$ Faculty of Engineering and the Environment, Aerodynamics and Flight Mechanics Group, University of Southampton, Southampton SO17 1BJ, UK

${ }^{3}$ Research Engineer, Computational Fluid Dynamics and Aeroacoustics Department, French Aerospace Lab, ONERA

* Corresponding Author, Tel.: +4423 80592172; Fax: +4423 80593190

Email address: pfj@isvr.soton.ac.uk (P. Joseph)
} 


\section{Introduction}

This paper presents the results of an experimental investigation into the use of leading edge serrations for reducing the broadband noise due to the interaction between turbulence and the leading edge $(L E)$ of aerofoils. Central to this paper is a parameter study in which the sensitivity of noise reduction are measured due to systematic variations in amplitude $(2 h)$ and wavelength $(\lambda)$ of a sinusoidal serrations profile. The main objective here is to identify experimentally the main geometric parameters that control the noise reduction performance of $L E$ serrations. For ease and economy of construction the experiments were performed on flat plates. Whilst extensive literature is now available on the use of serrations for reducing trailing edge noise, a detailed acoustical study on the use of sinusoidal $L E$ serrations on a flat plate are comparatively scarce. This paper provides the first detailed investigation on the noise reduction performance of flat plates with serrated leading edges subject to impinging turbulence. The effect of serrations on the $L E$ of flat plates are compared to the noise radiation due to a baseline flat plate with straight $L E$ to identify the effect of parameters $(\lambda$ and $h$ ) on noise reductions in terms of sound pressure and sound power levels.

There is also evidence that introducing $L E$ serrations on aerofoils can improve their aerodynamic performance. Collins [1] has observed that the presence of leading edge serrations on wings could improve the low-speed lift and stall performance of aircrafts during take-off and landing.

Bachmann et al. [2] have compared the noise due to the feathers of barn owl and pigeon, and highlighted the specific physiognomies of the owl's feathers on macroscopic as well as microscopic levels that are responsible for noise generation. They showed that the owl is quieter than the pigeon due to the presence of serrations at its leading edge and the fringes at the edges of each quill. They suggested that the mechanisms underlying quiet flight might ultimately be used for aerodynamic benefits leading to new wing designs. 
One of the first studies on the use of $L E$ serration to control aerofoil noise was by Soderman [3]. The noise characteristics of serrations mounted at the $L E$ of two different size rotors were investigated. The studies were conducted for various rotor speeds, blade angle as well as the geometry/location of serrations. He observed that high frequency noise was reduced with negligible effect on the low frequency noise. Further, it was noticed that the overall noise reductions varies from 4 to $8 \mathrm{~dB}$ for $1.52 \mathrm{~m}$ diameter rotor whereas it is up to 4 $\mathrm{dB}$ were for $2.59 \mathrm{~m}$ diameter rotor.

Hersh et al. [4] have showed the effectiveness of $L E$ serrations in reducing the narrow band vortex shedding noise emanating from stationary and rotating aerofoils. Noise reductions of between 4 to $8 \mathrm{~dB}$ were observed. They also showed that the $L E$ serrations reduces the tones generated by the periodic fluctuating forces near the trailing edge by forming vortices which alters the wake such that the velocity fluctuations changed from periodic to random.

Much more recently, Alex et al. [5] have investigated the effects of wavy leading edges on aerofoil-gust interaction noise. They showed that the main factor that determines noise reduction performance is the ratio of the leading edge amplitude to the wavelength of the incident gust. The calculations were based on periodic velocity fluctuations mainly in the vertical and streamwise directions which are responsible for the surface pressure fluctuation of an aerofoil. They found that significant noise reductions were achieved when the ratio of the leading edge amplitude to the gust wavelength exceeds about 0.3 . They found that the wavy leading edge causes a more rapid phase variation of pressure fluctuations along the $L E$ as compared to the straight leading edge, thereby leading to far field noise reductions.

Clair et al. [6] have recently performed a numerical and experimental investigation into the effect of sinusoidal leading edge serrations for the reduction of aerofoil-turbulence interaction noise. Sound power level reductions of about 3 to $4 \mathrm{~dB}$ were obtained for all flow 
velocities under consideration without affecting the aerodynamic performances. Predicted noise reductions were found to be greater than that measured at high frequencies (above 3- 4 $\mathrm{kHz}$ ), which was attributed to the spanwise gusts contribution being neglected in the computations.

Roger et al. [7] have also studied experimentally the sound generation due to the impingement of turbulent flow at the $L E$ of a NACA-12 aerofoil and its reductions using sinusoidal $L E$ serrations. They proposed a new analytical model for the noise reductions due to serrated leading edges by extending Amiet's theory [8]. The recent studies made by Haeri et al. [9] showed that noise reductions increase with increasing serration amplitude, thus corroborating the experimental findings reported by the authors of this paper [10]. Following this paper [10], the present paper provides a detailed experimental investigation into the aeroacoustic characteristics of the $L E$ serrated flat plates for various parameters $(\lambda, h)$ and its comparison with NACA 65 airfoils for the same set of leading edge serration parameters.

\subsection{Objectives of the current study}

The previous work discussed above demonstrates unequivocally that leading edge serrations are a potentially effective device for reducing broadband leading edge interaction noise. None of these studies, however, elucidate the dependence of noise reduction on the geometric parameters of the $L E$ serration, in particular their period $\lambda$, amplitude $h$ and dependence on mean flow velocity $U$. This paper provides the results of a parametric study aimed at understanding the effect of these various parameters on $L E$ serrations for producing aerofoil noise reductions. Preliminary numerical predictions are also included to support the experimental results presented in this paper. 


\section{Experimental set-up and procedure}

\subsection{Flat plate models}

For ease of manufacture and cost, experiments presented in this paper use flat plate aerofoils. We will show that whilst the noise reductions obtained from flat plate leading edge serrations are significantly greater than those achieved on realistic aerofoils for the same leading edge geometry, the essential physics is maintained and flat plate models are a useful approximation for determining optimal leading edge geometries. Three dimensional flow effects are of course absent from these flat plate measurements, which are anticipated to be significant only at high flow speeds.

A metallic flat plate of $2 \mathrm{~mm}$ thick [150 mm x $450 \mathrm{~m}$ ] was constructed by joining two flat plates each of $1 \mathrm{~mm}$ thick. This type of construction provides a slot at the leading edge of the flat plate for mounting the serration inserts cut from acrylic sheet of $2 \mathrm{~mm}$ thickness. The trailing edge of the flat plate was sharpened to eliminate vortex shedding noise. Acoustic measurements were made for various $L E$ serration geometries to quantify the effects of serration geometry on noise reductions. A schematic of the flat plate model is shown in Fig. 1 (a). In this study the serration geometry was restricted to sinusoidal profiles, which have been previously shown to be effective. The chord length of the $L E$ serrated (c) flat plate with amplitude $h$ and wavelength $\lambda$ is of the form

$$
c(r)=c_{o}+h \sin (2 \pi r / \lambda)
$$

where $c_{o}$ is the mean chord and $r$ is the spanwise distance. The profile of Eq. 1 ensures that the wetted area is maintained constant.

The noise power spectral density was measured for twenty serrated aerofoil geometries comprising all combinations of the sinusoidal serrations of different wavelengths $\left(\lambda / c_{o}\right)$ of $0.033,0.067,0.1$, and 0.133 and amplitudes $\left(h / c_{o}\right)$ of $0.033,0.067,0.1,0.133$ and 0.167 . Note 
that in this paper, for convenience, all serration lengths and periods will be normalised by the mean chord. However, there is no evidence to suggest that this non-dimensional parameter is important in determining noise reduction performance. The results were compared to the radiation from a baseline flat plate (un-serrated $L E$ ) and the sound power reduction obtained versus frequency. A schematic of the $L E$ serration showing the various parameters is shown in Fig. 1 (b). Photographs of the $L E$ serrated-flat plate and airfoil are shown in Figs. 1 (c) and 1 (d) respectively.
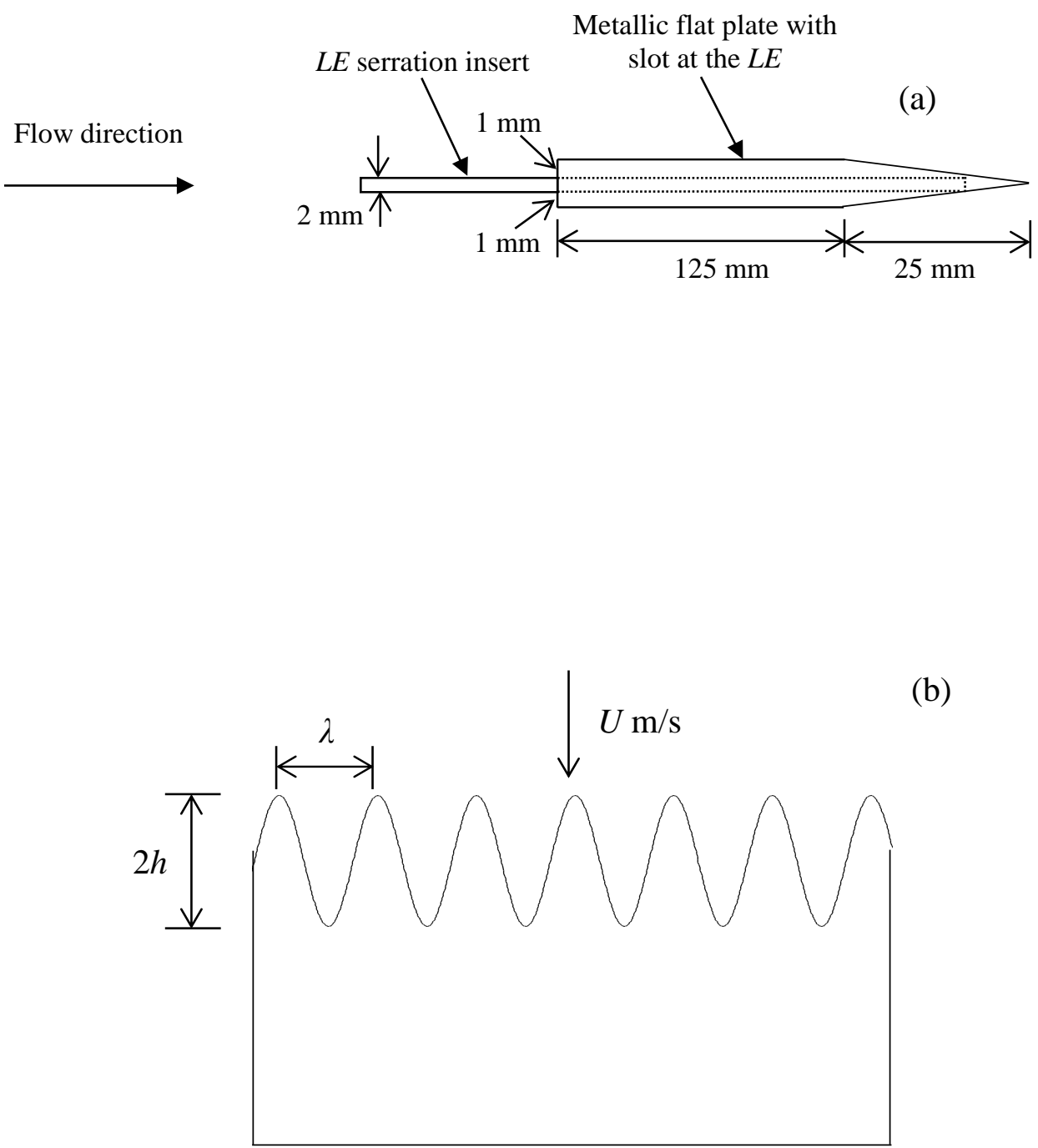

(b) 

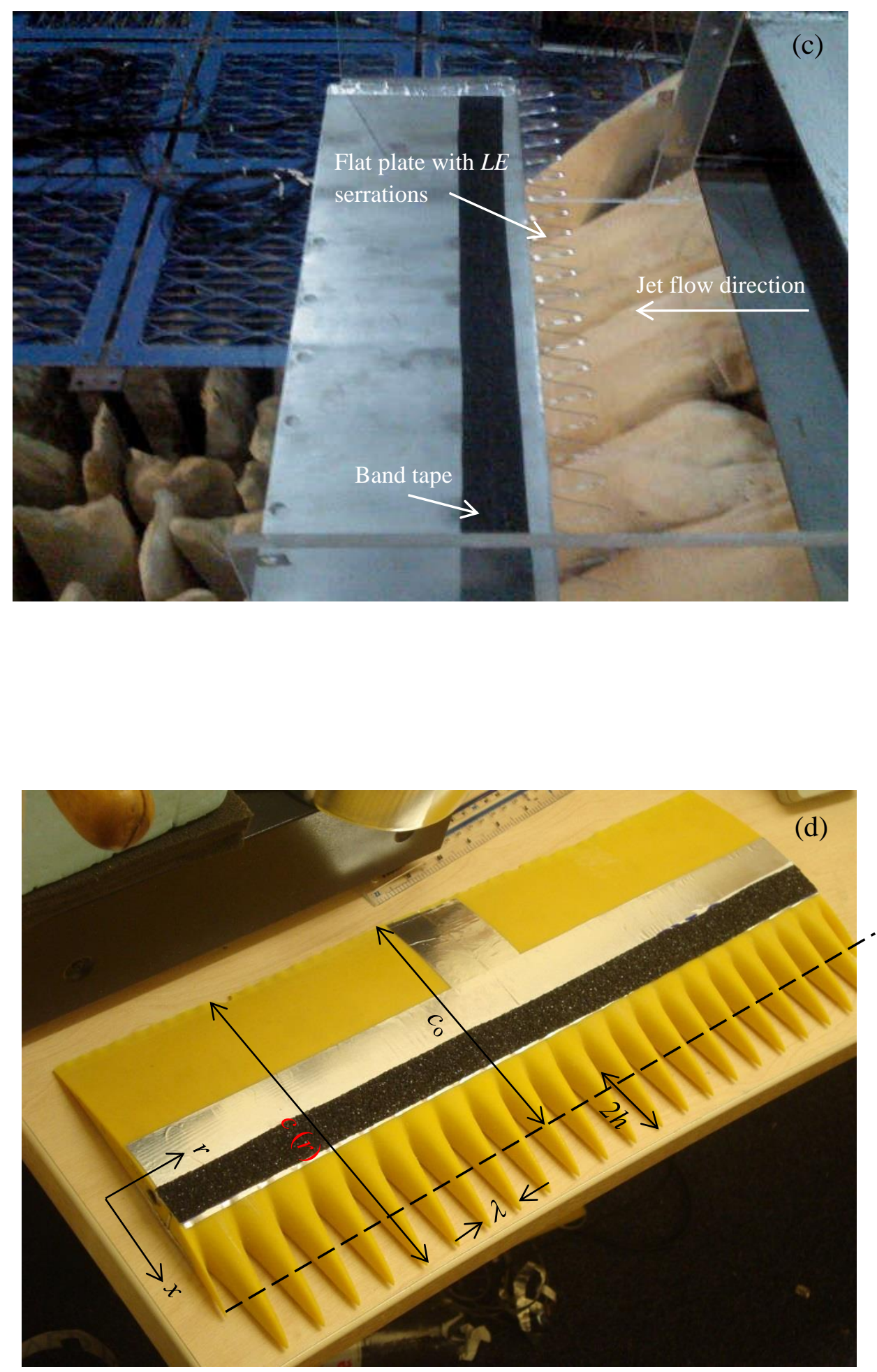

Fig. 1 (a) Flat plate model, (b) Schematic of smooth sinusoidal $L E$ serrations showing the parameters $h$ and $\lambda$, (c) A photograph of $L E$ serrated flat plate and jet nozzle inside the ISVR's anechoic chamber, (d) A photograph of the $L E$ serrated airfoil showing all the parameters (All dimensions are in $\mathrm{mm}$ ) 


\subsection{Jet test facility and instrumentations}

A photograph of the open-jet test facility inside the ISVR's anechoic chamber, with dimensions $8 \mathrm{~m} \times 8 \mathrm{~m} \times 8 \mathrm{~m}$, is shown in Fig. 2. A centrifugal fan mounted on the ceiling of the anechoic chamber, driven by a variable speed $110 \mathrm{~kW}$ motor, provides the flow. Fan noise is reduced by a 3-pass lined silencer. Air passes through a vertical duct on the wall and is then guided by a $90^{\circ}$ curved diffuser [11]. A second straight silencer tunnel that comprises a series of grids and honeycomb sections to ensure a quiet, uniform and low-turbulence flow. Finally, the air is passed through a nozzle of area ratio $25: 1$ to minimize the lateral velocity fluctuations. The height and width of the nozzle exit are 0.15 and $0.45 \mathrm{~m}$. The flat plate test models were held parallel to the jet flow direction by side plates mounted at the nozzle exit, to maintain two dimensional flow.

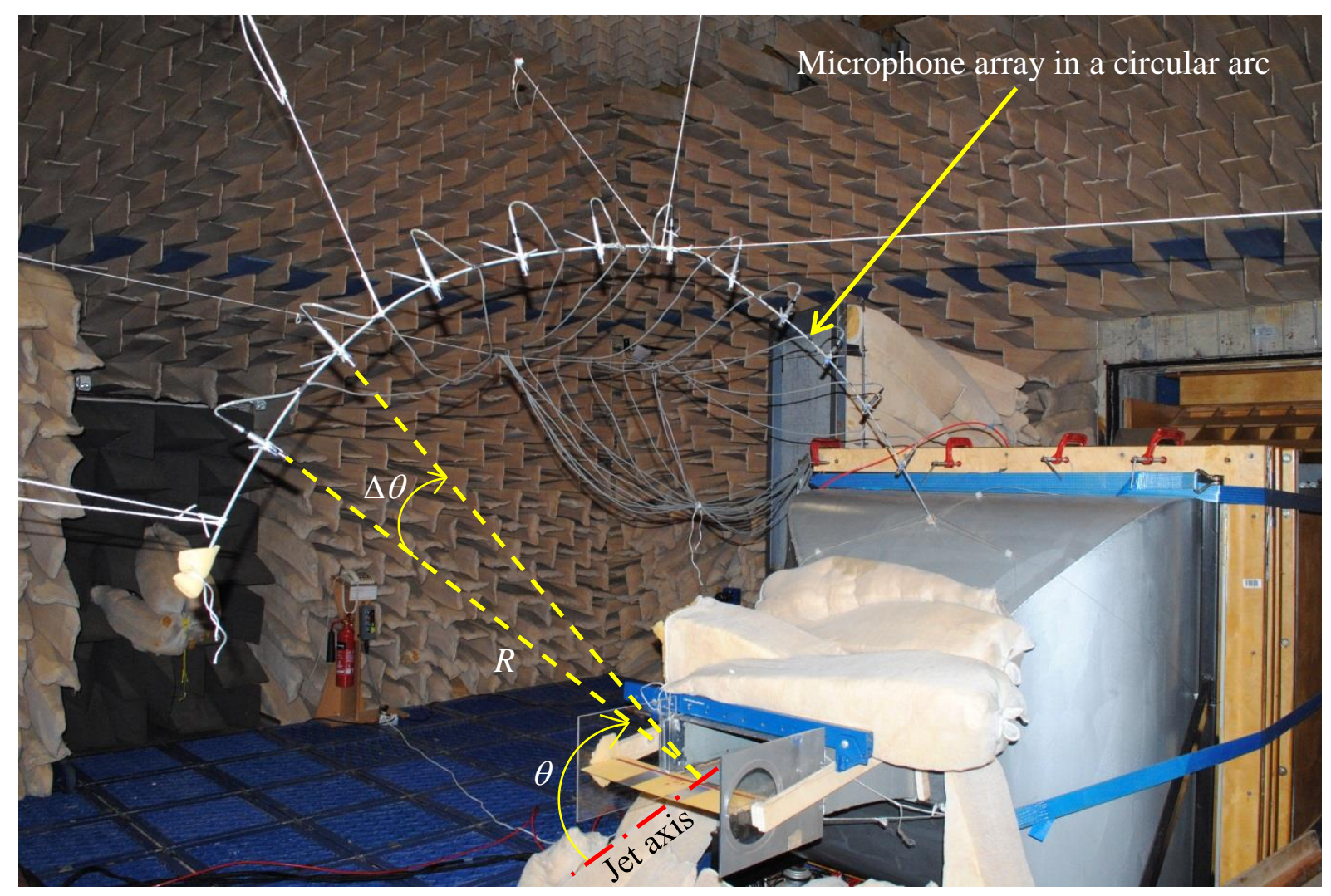

Fig. 2 Photograph of jet nozzle and test setup inside the ISVR's anechoic chamber (All dimensions are in $\mathrm{mm}$ ) 
Acoustic measurements were made by $11,1 / 2$ " condenser microphones (B\&K Model no: 4189-L-001) distributed over a circular arc at a radius of $1.2 \mathrm{~m}$ from the $L E$ of the flat plate. The array covered a range of emission angles $(\theta)$ between $40^{\circ}$ to $140^{\circ}$ measured relative to the downstream jet axis. Far field noise spectra were computed from time series of $10 \mathrm{~s}$ duration, sampled at $50 \mathrm{KHz}$, with a window size of 1024 data points corresponding to a frequency resolution of $48.83 \mathrm{~Hz}$. Acoustic data were recorded at the four mean flow velocities $(U)$ of 20,40, 60 and $80 \mathrm{~m} / \mathrm{s}$. In order to prevent instability noise from the trailing edge the flow near the $L E$ of flat plate was tripped using a rough band of tape on both suction and pressure sides to ensure fully developed turbulence in the boundary layer. The noise radiation is investigated in terms of sound pressure level spectrum $S P L$, defined in Eq. 2, and sound power level spectrum $P W L$ assuming cylindrical radiation, resembling more closely a line source than a point source is given in Eq. 4.

$$
\operatorname{SPL}(f)=10 \log _{10}\left(S_{p p}(f) / p_{r e f}^{2}\right)
$$

where $S_{p p}(f)$ is the power spectral density of pressure and $p_{\text {ref }}$ is $20 \times 10^{-6} \mathrm{~Pa}$.

$$
\begin{gathered}
S_{W}(f)=\left(\frac{L R}{\rho a}\right)\left\{\sum_{i=1}^{i=N-1}\left(\frac{S_{p p}\left(\theta_{i}\right)+S_{p p}\left(\theta_{i+1}\right)}{2}\right) \Delta \theta\right\} \\
P W L(f)=10 \log _{10}\left(W(f) / W_{r e f}\right)
\end{gathered}
$$

where, $S_{W}(f)$ is the spectral density of the sound power radiated between the radiation angles $\left[40^{\circ}\right.$ to $\left.140^{\circ}\right], W_{\text {ref }}=10^{-12} \mathrm{~W}$ and $S_{p p}\left(\theta_{i}\right)$ is the acoustic pressure PSD (Power Spectral Density) measured at measurement angle $\theta_{i}$ and $N$ is the number of microphones [11], $L$ is the span of the flat plate [450 mm], $R$ is radius of the microphone array $(1.2 \mathrm{~m}), \Delta \theta$ is the angle between the two adjacent microphones (10 degrees), $\rho$ is the density of the ambient air in $\mathrm{kg} / \mathrm{m}^{3}$, and $a$ is the speed of sound in $\mathrm{m} / \mathrm{s}$. 


\subsection{Characterization of the jet with turbulence grid}

A good approximation to homogeneous and isotropic turbulence was generated using a biplanar turbulence grid with overall dimensions of $630 \times 690 \mathrm{~mm}^{2}$ located in the contraction part of the nozzle (Fig. 3). Hot wire anemometry was used to measure and characterize the mean and unsteady flow at the location of the airfoil's leading edge i.e., $145 \mathrm{~mm}$ from the nozzle exit. The turbulence integral length scale was estimated by comparison of the measured spectrum with the Von Karman spectrum for longitudinal isotropic turbulence by Pope [12]. The comparison with Von Karman spectrum would be helpful in order to characterize the flow with controlled turbulent fluctuations. The experiments and theory are in excellent agreement, hence confirming that the turbulence generated in the test-rig was isotropic to a good degree [black curve]. The average turbulence intensity level for the grid and the integral length scale at $145 \mathrm{~mm}$ from the nozzle exit was $2.5 \%$ and $6 \mathrm{~mm}$ and are maintained constant in the current set of measurements.

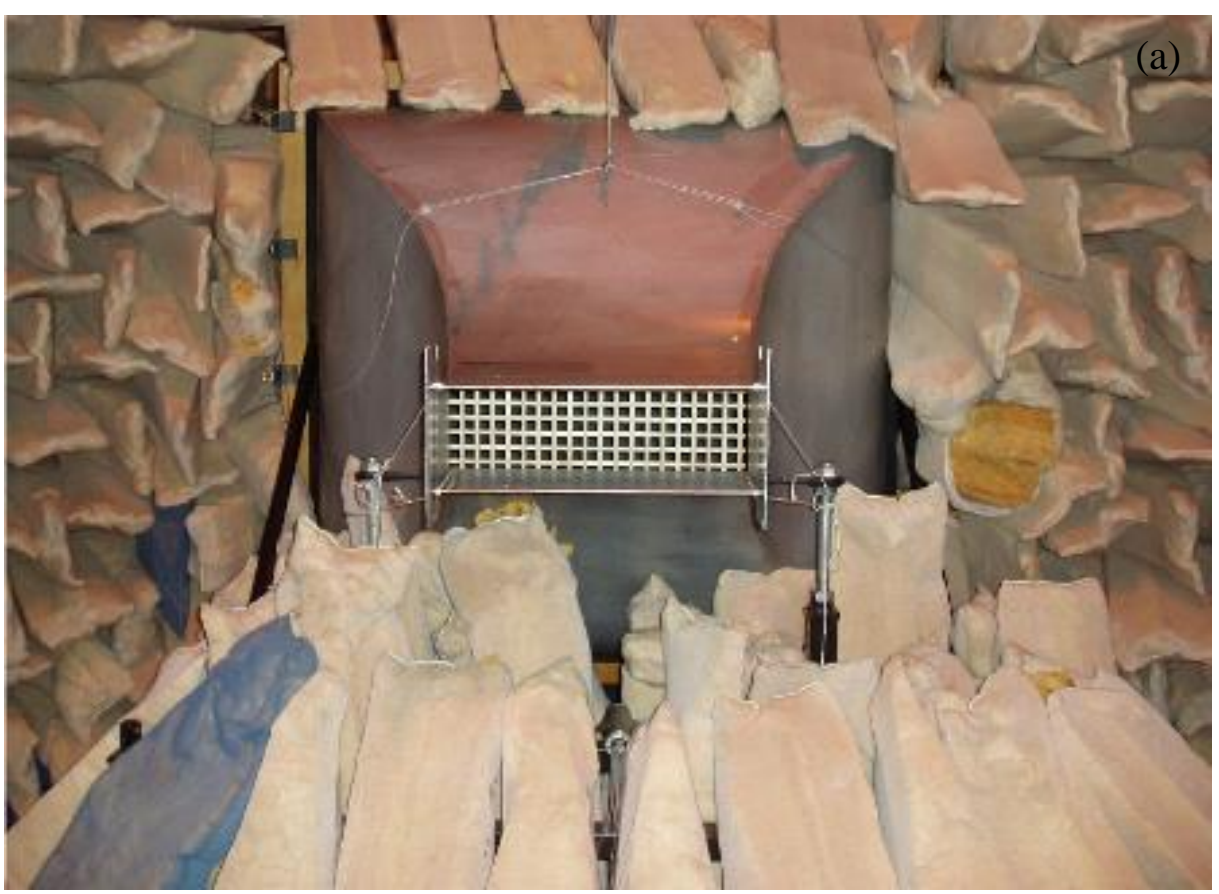




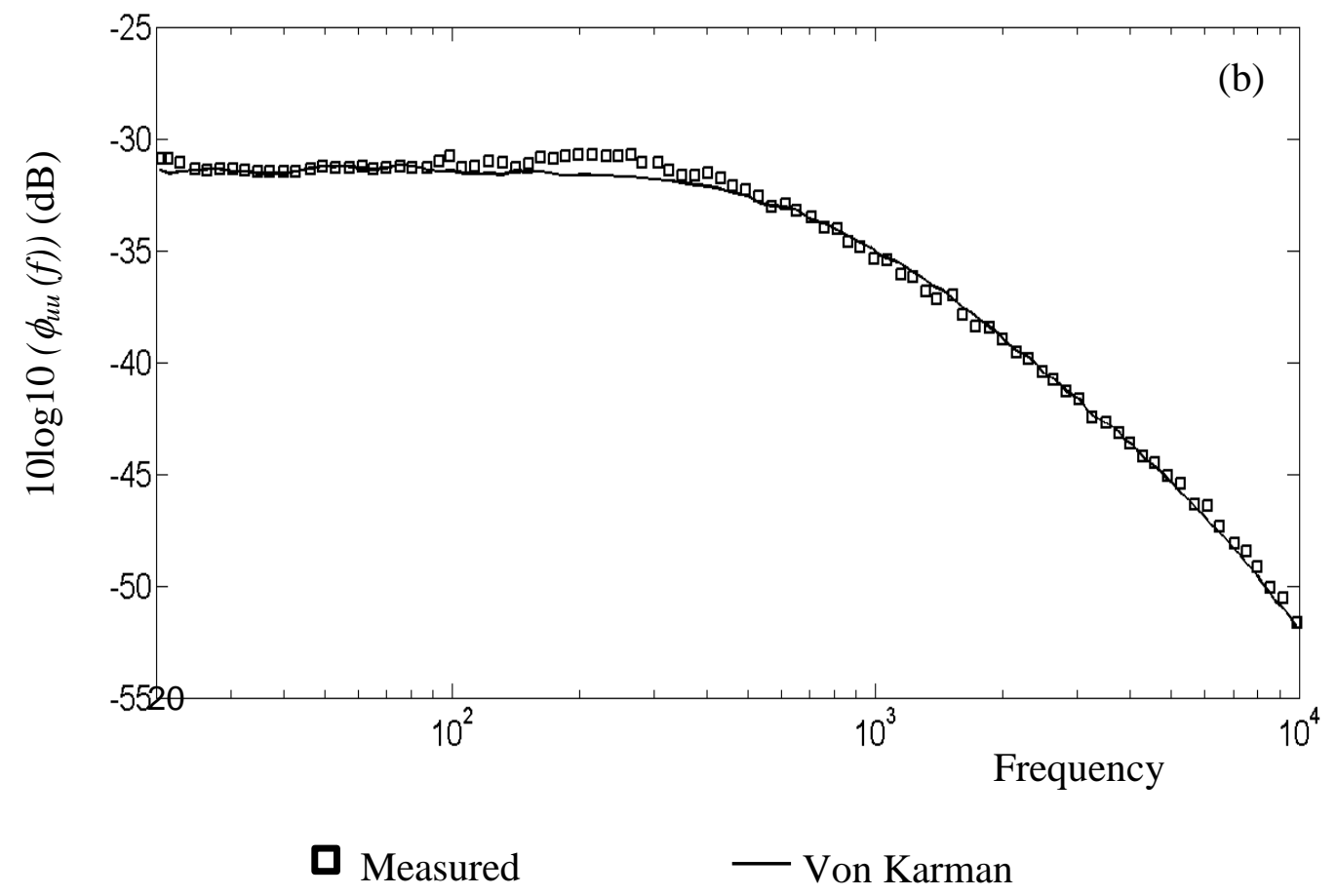

Fig. 3 (a) Turbulence grid located in the contraction section of the nozzle, (b) Comparison of the measured streamwise velocity spectrum $\left(\phi_{u и}\right)$ against Von Karman model for longitudinal isotropic turbulence, at $U=40 \mathrm{~m} / \mathrm{s}$

\section{Results and discussions}

\subsection{Spectral characteristics}

\subsubsection{Acoustic spectra comparison of baseline and serrated-flat plates}

Although the paper highlights the reduction of leading edge interaction noise, it is very difficult to eliminate the trailing edge self-noise from the overall noise radiation from the aerofoil. It has been shown that the leading edge interaction noise is the dominant noise source as compared to the trailing edge self-noise when the aerofoil is subjected to the turbulence stream generated by our grid. The influence of self-noise is generally observed at high frequencies and this has been illustrated in section 3.3. All the noise reductions shown in this paper are based on measurements of the total noise radiated (i.e., the sum of leading edge interaction noise and trailing edge self-noise) from the aerofoil. 
By way of initial validation of the measurement method, and to establish the baseline noise spectra, the measured acoustic spectra due to interaction with grid generated turbulence by a flat plate with sharp trailing edge (red curve) is compared with those predicted using Amiet's theory [8] in Fig. 4 (red dashed curve). The assumption of homogeneous and isotropic turbulence as mentioned above is essential to allow noise predictions to be made using both the Amiet theory and numerical prediction obtained from the Euler solution. Good general agreement is observed, with the characteristic oscillations (interference peaks) closely matching up to about $9 \mathrm{kHz}$. The mismatch observed between prediction and measurements at low frequency is due to the dominance of jet noise at very low frequencies and also due to grid-generated narrow-band vortex shedding and its interaction with aerofoils. Background noise measurements at the same velocity with turbulence grid are also plotted in Fig. 4 which can be seen to be substantially below the total noise radiated from the aerofoil. The shedding tone due to grid is observed in the background noise spectra coincide with the tone in interaction noise which also explains the mismatch between predictions and measurements at low frequency.

The acoustic spectra following the introduction of the $L E$ serrations is also shown in Fig. 4 at $U=60 \mathrm{~m} / \mathrm{s}$ for all $L E$ amplitudes $\left(h / c_{o}\right)$ at a wavelength $\left(\lambda / c_{o}\right)$ of 0.067 . Significant noise reductions (greater than about $3 \mathrm{~dB}$ ) are confined to high frequencies (500 $\mathrm{Hz}$ to $8 \mathrm{kHz}$ ) with negligible noise reductions below about $0.5 \mathrm{kHz}$ in this example. Figure 4 indicates that the level of noise reduction increases as the serration amplitude $h$ is increased. A noteworthy feature of the radiation spectra obtained with serrations is that oscillations that appear in the pressure spectrum obtained with the sharp edge (red curves) are much reduced. This phenomenon is because when $L E$ serrations are introduced the pressure jump across the chord and along the span is no longer coherent due to scattering of the pressure at the serrated leading edge. Peaks in the spectrum due to interference across the chord are therefore 
suppressed and oscillations in the radiation spectrum arising from coherent radiation across the chord are therefore much reduced. Greatest noise reductions in the pressure spectra therefore appear at the frequencies very roughly corresponding to the peaks in the sharp edge flat plate radiation spectra, which at low Mach number $\left(\sqrt{1-M^{2}} \sim 1\right)$ may be observed to occur at $f c_{\mathrm{o}} / a=n+1 / 4$, where $n$ is an integer, as shown in a later section. However, no such observation can be found for the sound power which does not oscillate with frequency.

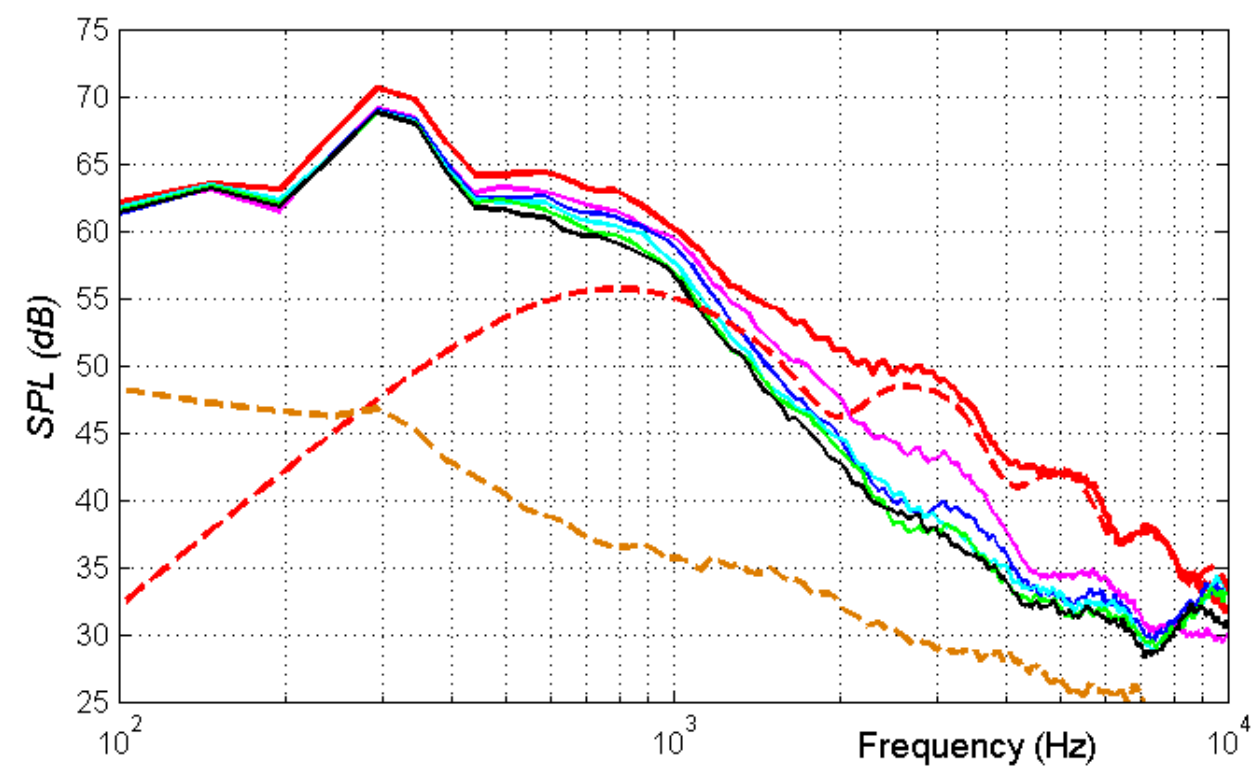

$\begin{array}{lll}\text { - Measured baseline flat plate } & \text {---- Predicted baseline flat plate } \quad-h / c_{o}=0.033 \\ -h / c_{o}=0.067-h / c=0.1 \quad-h / c=0.133 \quad-h / c=0.167 \quad-- \text { Background noise }\end{array}$

Fig. 4 Typical sound pressure spectra comparison of baseline and serrated-flat plates at $U=$ $60 \mathrm{~m} / \mathrm{s}$ for various amplitudes $\left(h / c_{o}\right)$ at a wavelength $\left(\lambda / c_{o}\right)$ of 0.067

3.1.2 Sound power level spectra and sound power reduction levels ( $\triangle P W L s)$ of flat plate and NACA-65 aerofoil

In this paper, noise measurements were made on flat plate aerofoils for ease and cost of construction. We now compare in Fig. 5 (a) the radiation spectra from flat plate experiments with those obtained on a NACA-65 aerofoil, with the identical serration parameters of $\lambda / c_{o}=$ $0.067, h / c_{o}=0.067$ at a velocity $(U)$ of $40 \mathrm{~m} / \mathrm{s}$. A photograph of the serrated airfoil showing 
the parameters such as $\lambda, h, c_{o}$ and the span-wise sinusoidal modulation $c(r)$ are shown in Fig. 1 (d). The serrated airfoil was manufactured according to,

$$
y=\left\{\begin{array}{l}
f\left(x / c_{o}\right) \quad 0<x / c_{0}<2 / 3 \\
f(x / c(r)) \quad 2 / 3 \leq x / c(r) \leq 1
\end{array}\right.
$$

where $f(X)$ defines the NACA-65 profile at each plane $r$ as a function of normalised chord $(0$ $<X<1$, where $X=0$ and $X=1$ are at the trailing and leading edges respectively), $c(r)=c_{o}+$ $h \sin (2 \pi r / \lambda)$, as already mentioned in Eq. 1

The sound power level spectra for the baseline (un-serrated) flat plate (solid red curve) in Fig. 5 is higher than for the baseline NACA-65 aerofoil (solid blue curve), with the noise reduction increasing as frequency increases. In the case of flat plates we observe a slight increase in sound power level at high frequencies $(8-9 \mathrm{kHz})$ compared to the baseline. This may be due to an increase in self-noise as a result of flow separation from the corners of the flat plate leading edge whereas no corresponding increase in self-noise is observed for the aerofoils. The effect of aerofoil thickness on interaction noise is well documented. The lower broadband noise radiation for thick aerofoils can be attributed to a local slowing of the turbulent gust as it approaches the leading edge close to the stagnation region.

A comparison of sound power reduction levels $(\triangle P W L)$ versus frequency for the flat plate and the NACA-65 aerofoil is shown in Fig. 5 (b). The maximum noise reduction for the flat plate and aerofoil are around 9 and $7 \mathrm{~dB}$ at frequencies of about 2.4 and $1.8 \mathrm{kHz}$ respectively. In general, it is observed that the flat plates provide higher levels of noise reduction compared to the NACA-65 aerofoil over the range of frequencies investigated (Fig. $5(b))$. 

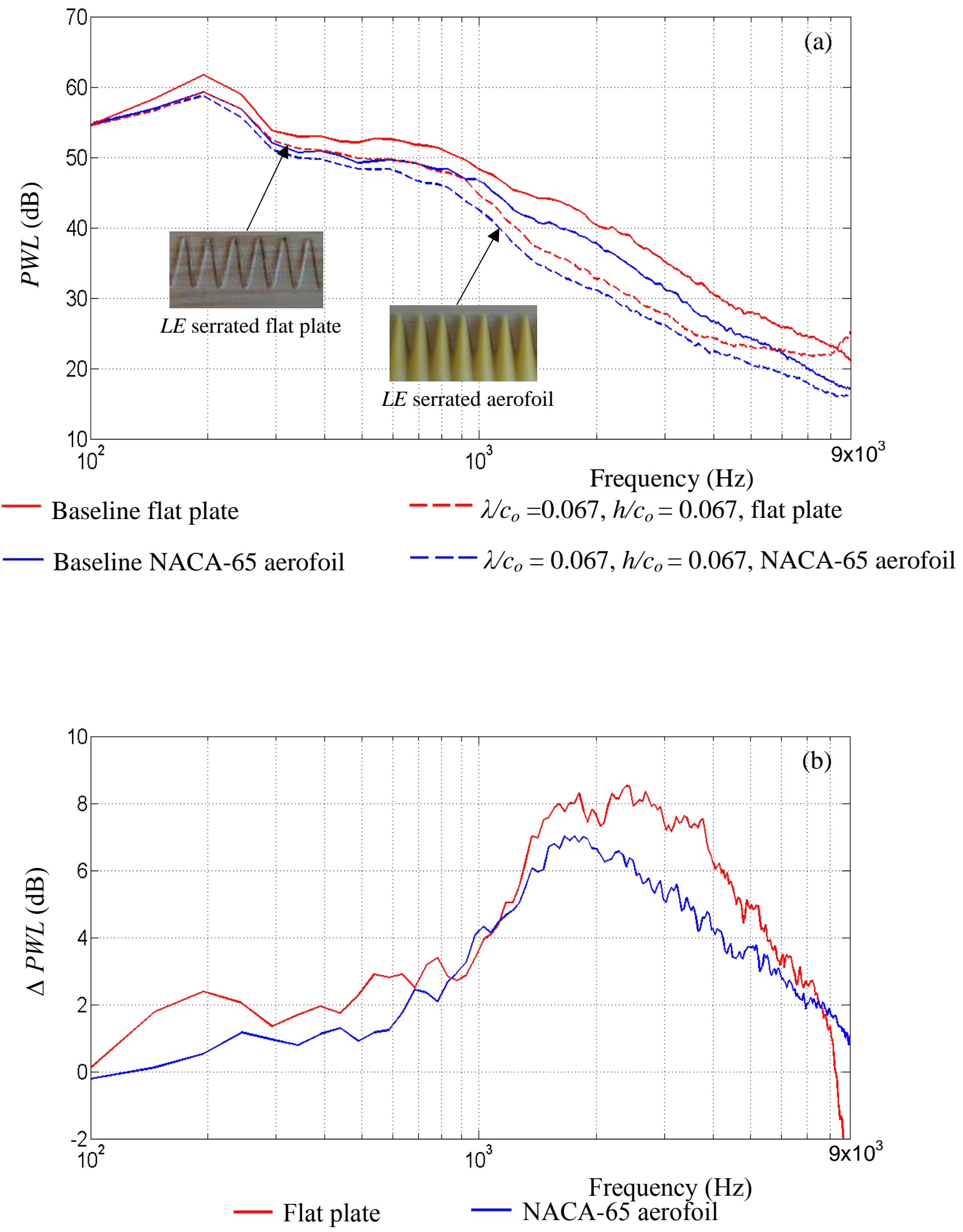

Fig. 5 (a) Sound power level spectra comparison of baseline flat plate and serrated flat plate $\left(\lambda / c_{o}=0.067, h / c_{o}=0.067\right)$ with baseline NACA-65 aerofoil and serrated NACA-65 aerofoil $\left(\lambda / c_{o}=0.067, h / c_{o}=0.067\right)$ at $U=40 \mathrm{~m} / \mathrm{s}$, (b) Sound power reduction levels $(\triangle P W L)$ comparison of flat plate aerofoil and NACA-65 aerofoil at $U=40 \mathrm{~m} / \mathrm{s}$ 


\subsection{Sound power level characteristics}

\subsubsection{Effect of mean jet velocity of noise reduction levels}

The sound power reduction levels $(\Delta P W L)$ of smaller $\left(h / c_{o}=0.067\right)$, and larger amplitude, serration $\left(h / c_{o}=0.167\right)$ at $\lambda / c_{o}=0.067$ for different jet velocities $(U)$ are compared in Fig. 6 . No significant variation in the noise reductions are observed for all jet velocities up to $f c_{d} / U$ of 5 in both amplitude serrations. The maximum noise reductions are observed to occur in the high frequency range $5<f c_{o} / U<15$ for all jet velocities in both amplitude serrations. In general, noise reductions at high frequencies are observed to increase for both amplitude serrations as the jet velocity increases. The increase in noise at high frequencies is due to the increase of self-noise from the serrated flat plates compared to the baseline. This may be due to changes in the boundary layer characteristics due to the leading edge serrations. At higher frequencies the noise reductions versus frequency show oscillatory behaviour at all jet velocities. Thus, there appears to be an optimum frequency where maximum noise reduction occurs. Both the serration amplitudes show similar trends for all jet velocities even though their maximum noise reductions differ slightly (i.e., $9 \mathrm{~dB}$ in case of smaller amplitude serration and $11 \mathrm{~dB}$ for larger amplitude ones). Thus, the comparison clearly shows that the $L E$ amplitude is the most important parameter in providing higher noise reductions for a particular jet velocity. Therefore, the sound power reductions for a fixed non-dimensional frequency $\left(f c_{o} / U\right)$ is observed to be weakly dependent upon flow velocity.

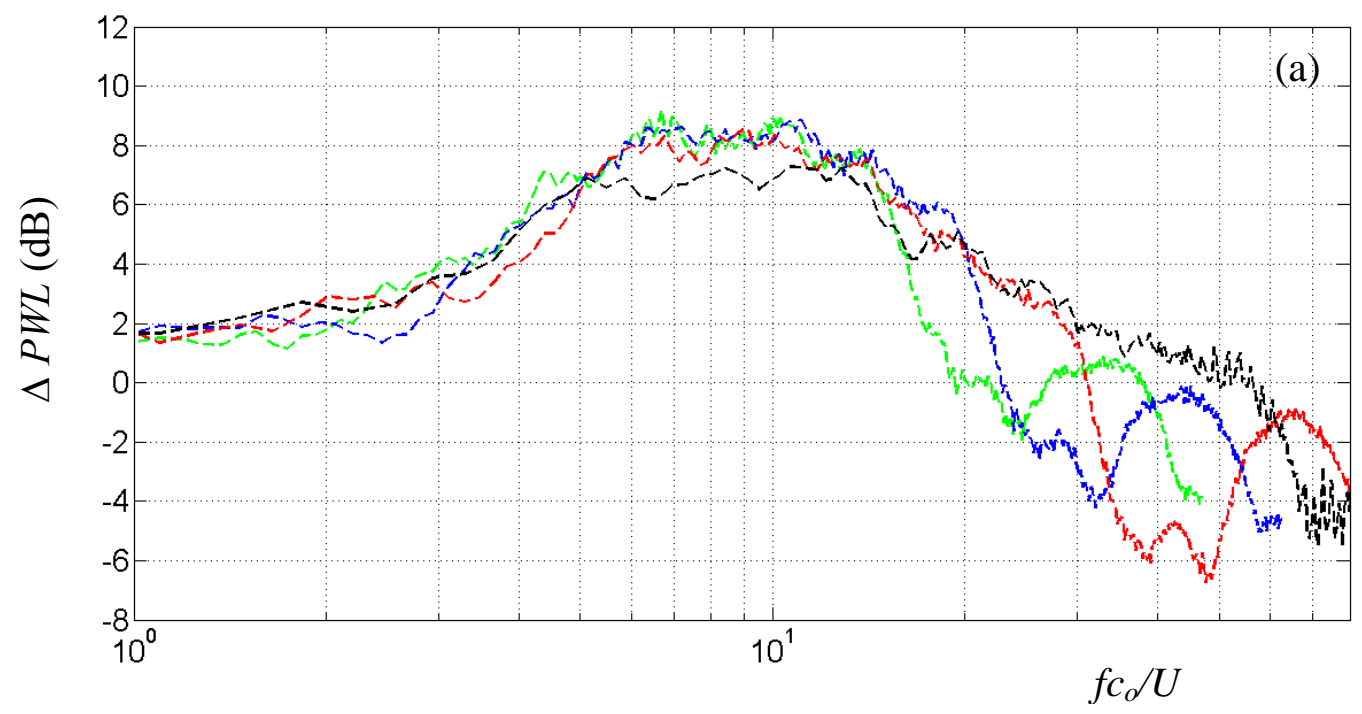




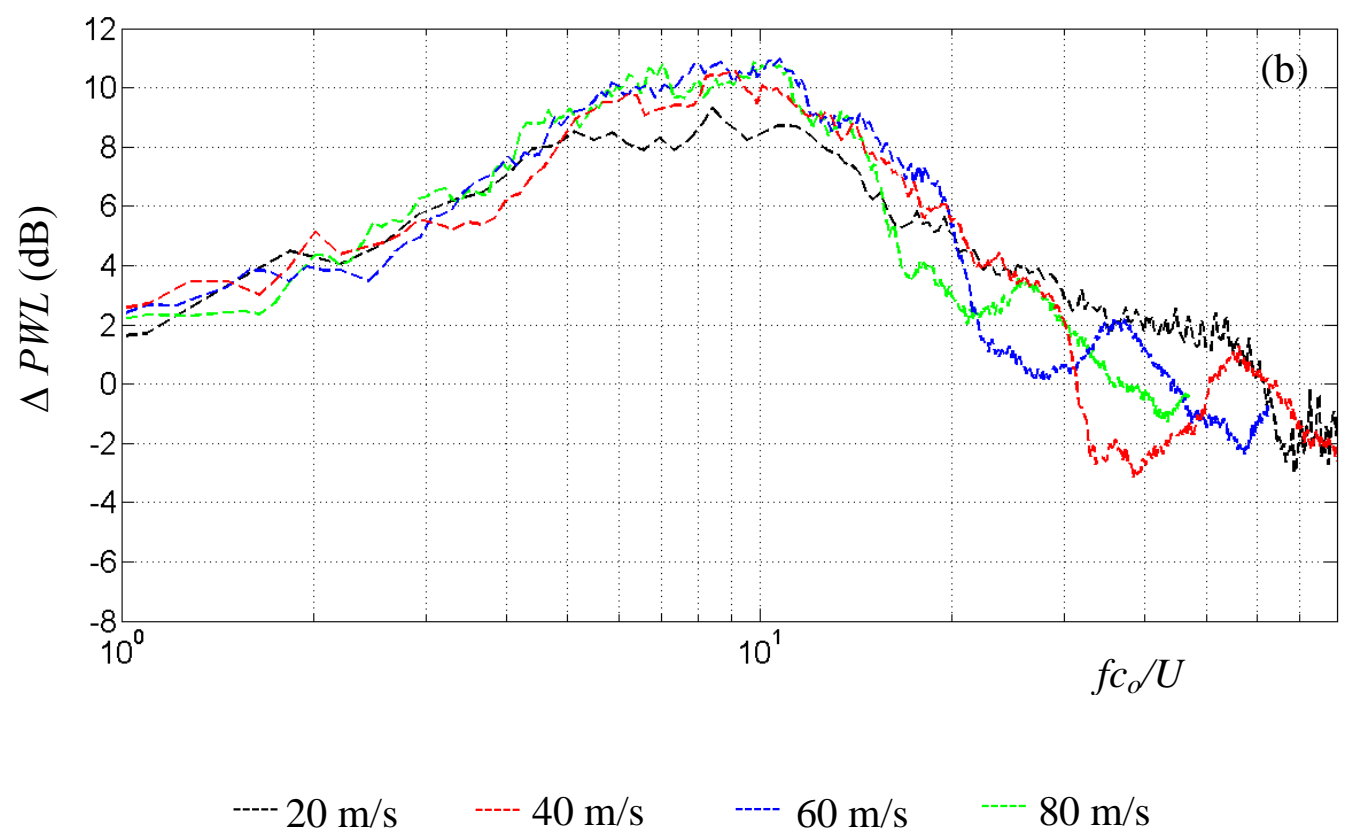

Fig. 6 Sound power reduction level $(\triangle P W L)$ comparison of (a) small amplitude serration $\left(\lambda / c_{o}=0.067, h / c_{o}=0.067\right)$, (b) large amplitude serration $\left(\lambda / c_{o}=0.067, h / c_{o}=0.167\right)$ for different jet velocities

\subsubsection{Effect of LE amplitude on Sound power reduction levels ( $\triangle P W L s)$}

The variation of sound power level reduction $(\Delta P W L)$ versus non-dimensional frequency $\left(f c_{o}\right.$ $/ U$ ) at $U=60 \mathrm{~m} / \mathrm{s}$ are shown in Figs. 7 (a-d) for different $L E$ amplitudes $\left(h / c_{o}\right)$ and different $\lambda / c_{o}$ values. The reduction in sound power level $(\triangle P W L)$ is generally observed to increase with increasing $L E$ amplitude $\left(h / c_{o}\right)$ for all the values of $\lambda / c_{o}$ values studied. A maximum noise reduction of about 12 and $10 \mathrm{~dB}$ are observed for large $h / c_{o}$ value of 0.167 at $\lambda / c_{o}$ values of 0.033 and 0.133 . In general, it is observed in Fig. 7 at smaller $f c_{o} / U$ values from 1 to 3 , the effect of serration amplitude becomes significant as the $\lambda / c_{o}$ value increases from 0.033 to 0.133. The relatively large reductions in sound power levels observed at higher $L E$ amplitude are likely to arise from mutual destructive interference of the far-field noise as a result of strong destructive interference of the acoustic pressures radiating along the serrated leading edge. The explanation for the noise reductions due to leading edge serrations has been shown 
to be due to destructive interference between the radiations from different parts of the leading edge serration, as shown by Haeri et al. [9], a co-author of this paper. It clearly demonstrates through simulation that a significant phase difference is observed between the unsteady pressures at various locations along the serrated leading edge compared to baseline case, leading to reduced radiation in the far field.
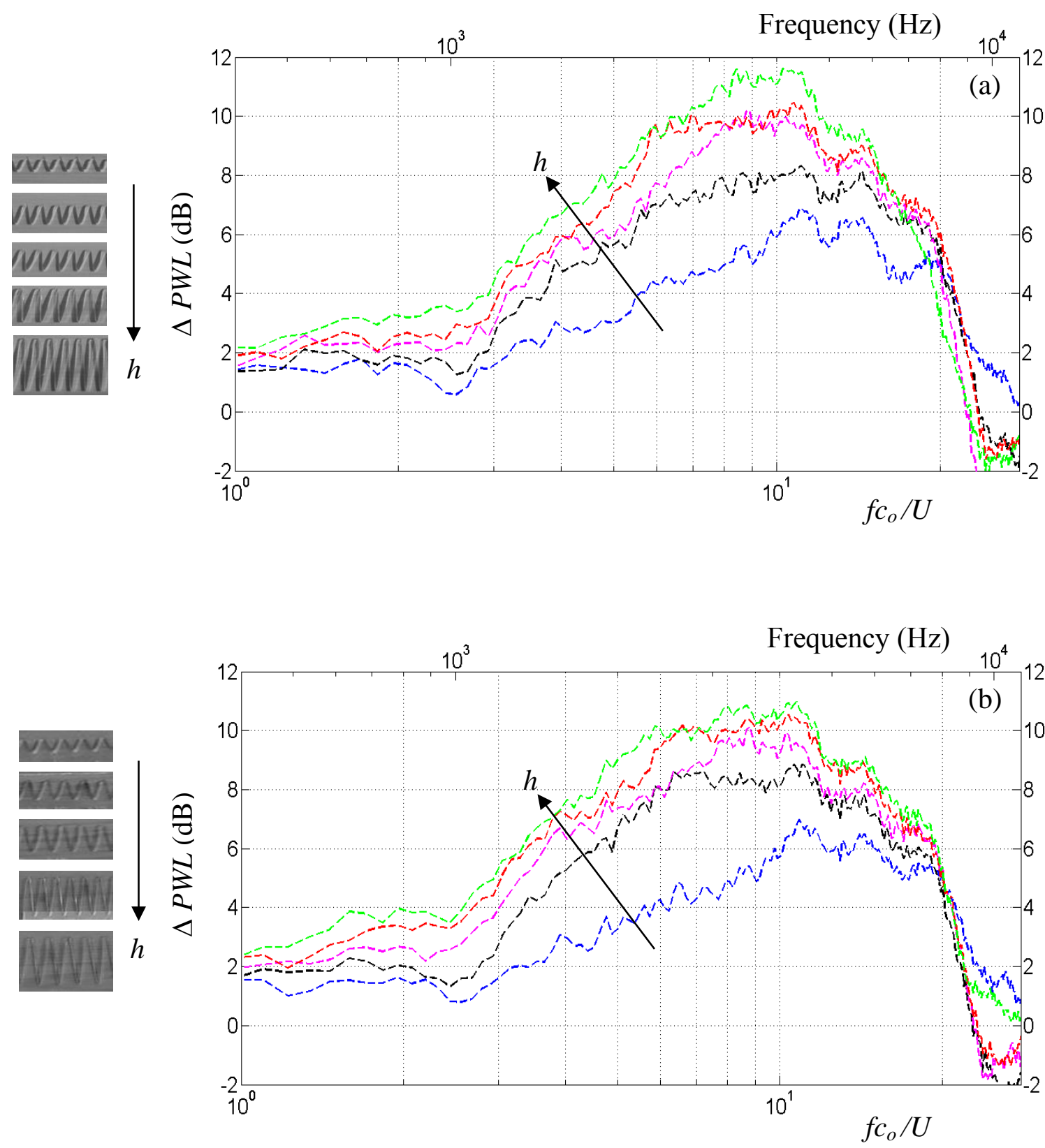

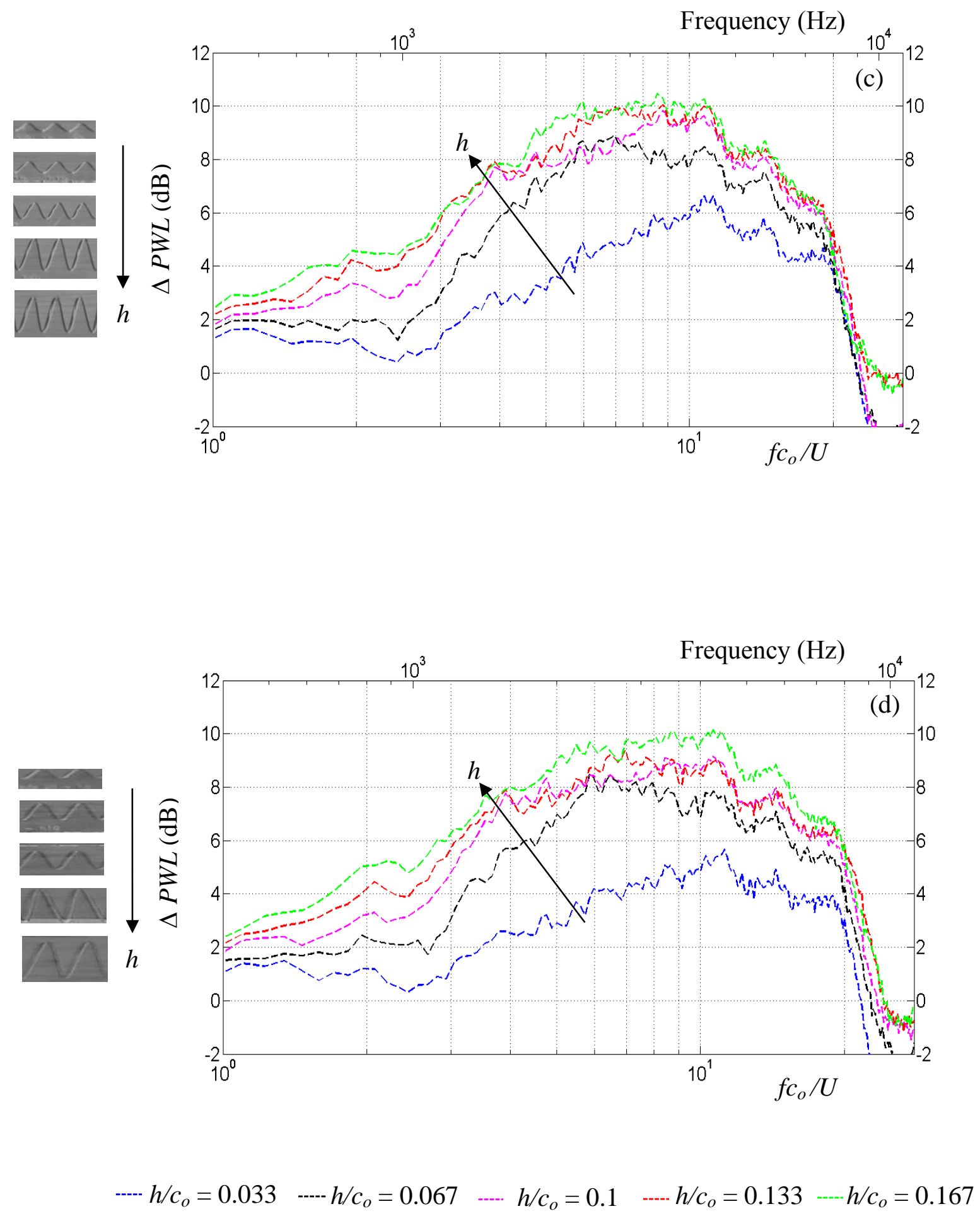

Fig. 7 Variation of sound power reduction levels $(\triangle P W L)$ with non-dimensional frequency at $U=60 \mathrm{~m} / \mathrm{s}$ for various amplitudes $\left(h / c_{o}\right)$ at wavelengths $\left(\lambda / c_{o}\right)$ of (a) 0.033 , (b) 0.067 , (c) 0.1 , (d) 0.133 


\subsubsection{Overall Sound power reduction levels $(\triangle O A P W L)$ of serrated-flat plates}

As shown in Figs. $6 \& 7$, the noise reductions are strong functions of non-dimensional frequency $\left(f c_{o} / U\right)$ and serration amplitudes. In the present section, the overall sound power levels for both serrated and un-serrated-flat plates were computed by integrating the sound power level over the frequency band $500 \mathrm{~Hz}$ to $8 \mathrm{kHz}$ and then used to compute the reduction in sound power level $\triangle O A P W L$ over this bandwidth.

The variation in overall sound power reduction level $(\triangle O A P W L)$ versus $L E$ amplitudes $h / c_{o}$ for various $U$ values are shown in Figs. 8 (a-d). In each figure noise reductions are shown as a function of $h / c_{o}$ on a logarithmic scale, for the different values of $\lambda / c_{o}$. For all the $\lambda / c_{o}$ values studied here $\triangle O A P W L$ increases logarithmically as the amplitude of the $L E$ serration $h / c_{o}$ increases. This suggest that the power reduction is inversely proportional to $h^{n}$ where $n$ is some frequency dependent constant. The reason for this dependence is currently unclear. However, it suggests that increasing serration amplitudes produces progressively smaller increases in noise reduction. The $\triangle O A P W L$ of NACA-65 airfoils at $h / c_{o}=0.067$ and $h / c_{o}=0.133$, where $\lambda / c_{o}=0.067$ in both cases are also shown in Fig. 8 for comparison. Figure 8 clearly shows that the serrated $L E$ integrated into a three dimensional aerofoil generally provides less noise reduction compared to those in flat plates. The likely reason that flat plate serrations offer superior noise reductions is that all the aerodynamic sources are situated in the plane of the flat plate leading to high levels of destructive interference in the far field radiation. For the NACA-65 airfoil, however, the sources are distributed over the 3D geometry of the serrated airfoil, leading to imperfect destructive far field interference. Furthermore, 3D flow features may also act as secondary noise sources that may mask the noise reductions provided by the $L E$ serrations. However, the trends of the noise reductions with serration amplitudes are identical in both cases. 
In general, it is seen that similar to $L E$ serrated flat plates, the serrated aerofoil also show diminishing noise reduction characteristics with increasing jet velocity. The reason for this will be discussed in the blow down section.
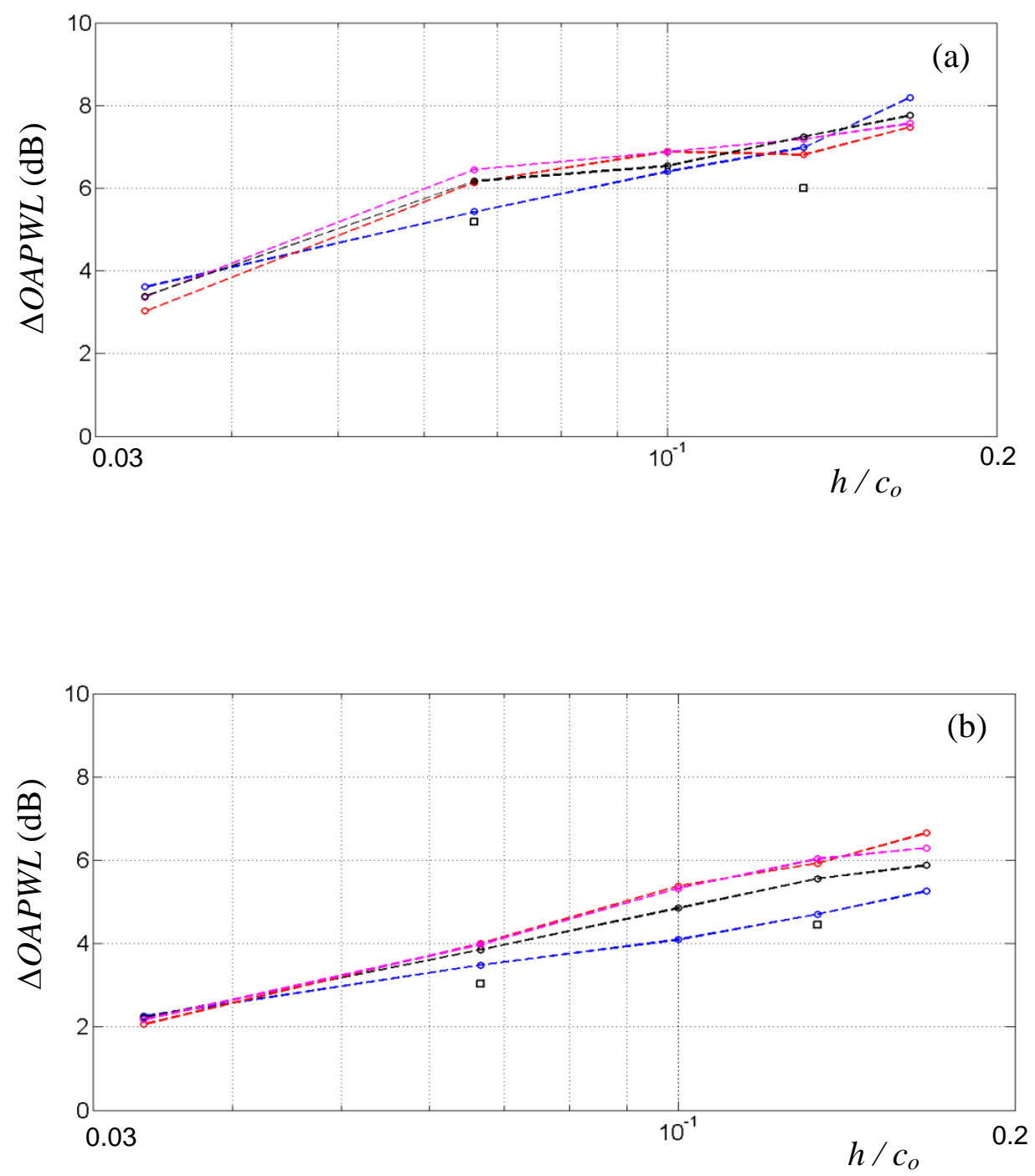

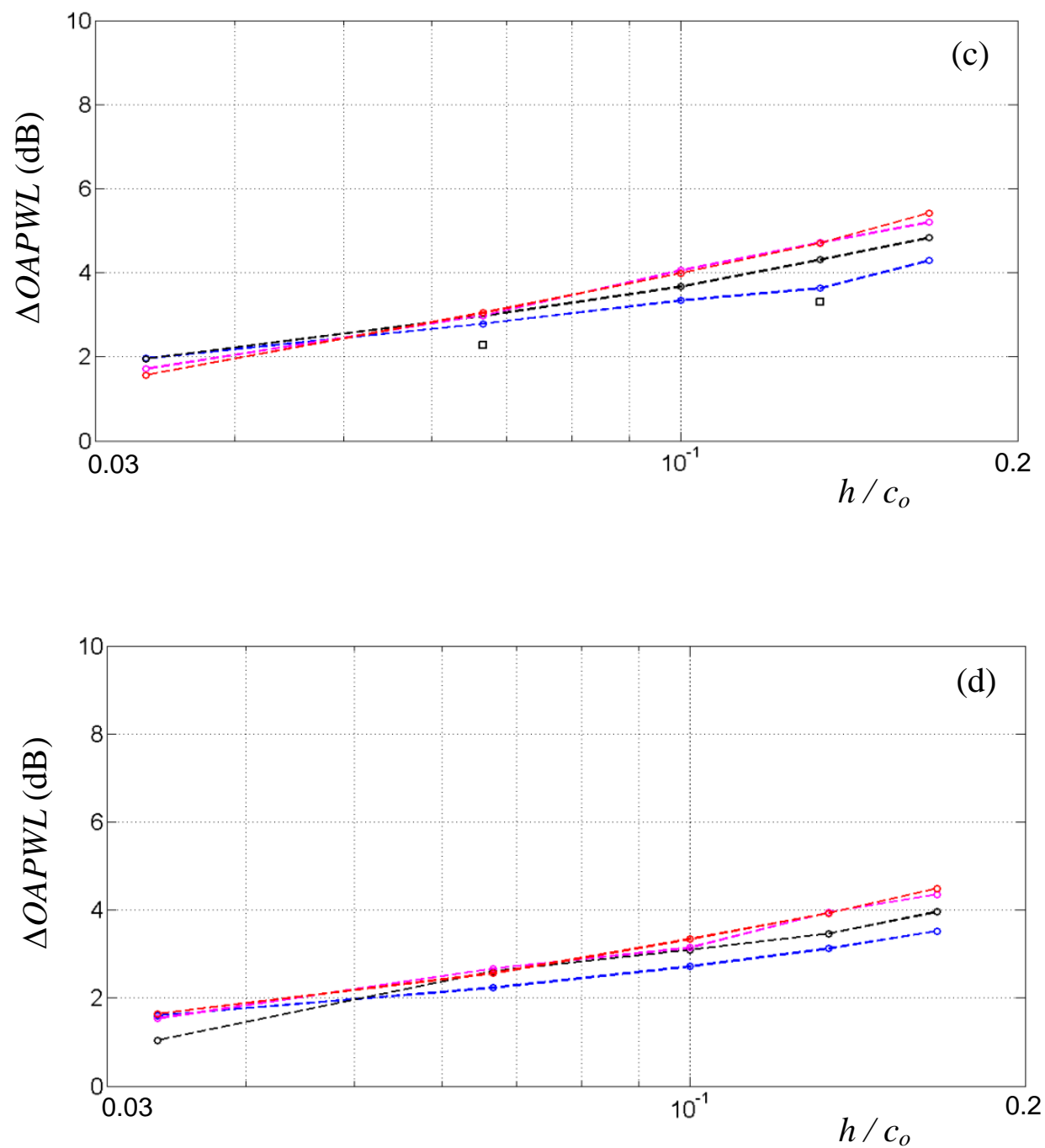

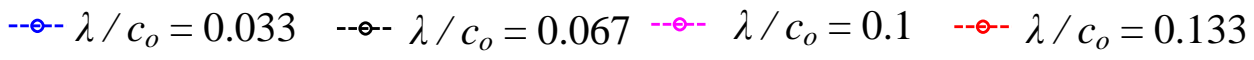

口 NACA 65 airfoil at $\lambda / c_{o}=0.067$

Fig. 8 Variation of $\triangle O A P W L$ with $h / c_{o}$ for various $U$ values of (a) $20 \mathrm{~m} / \mathrm{s}$, (b) $40 \mathrm{~m} / \mathrm{s}$, (c) 60 $\mathrm{m} / \mathrm{s}$, (d) $80 \mathrm{~m} / \mathrm{s}$ 
The variation of $\triangle O A P W L$ with $h / c_{o}$ at smaller and larger $\lambda / c_{o}$ values of 0.033 (dashed lines) and 0.133 (solid lines) are shown in Fig. 9 for all $U$ values. It clearly shows the decrease in overall noise reductions with increase in jet velocity as mentioned earlier (Fig. 8). Furthermore, a striking feature observed in Fig. 9 is that the serrations with larger $\lambda / c_{o}$ values, (i.e., wider serrations) provide higher overall noise reduction levels at higher $L E$ amplitudes and jet velocities $(40 \mathrm{~m} / \mathrm{s}, 80 \mathrm{~m} / \mathrm{s})$. Thus, wider and longer serrations achieve higher overall noise reduction levels than narrower and shorter serrations. This behaviour is contrary to that observed for trailing edge serrations where noise reductions improve as the serration period $\lambda$ is reduced.

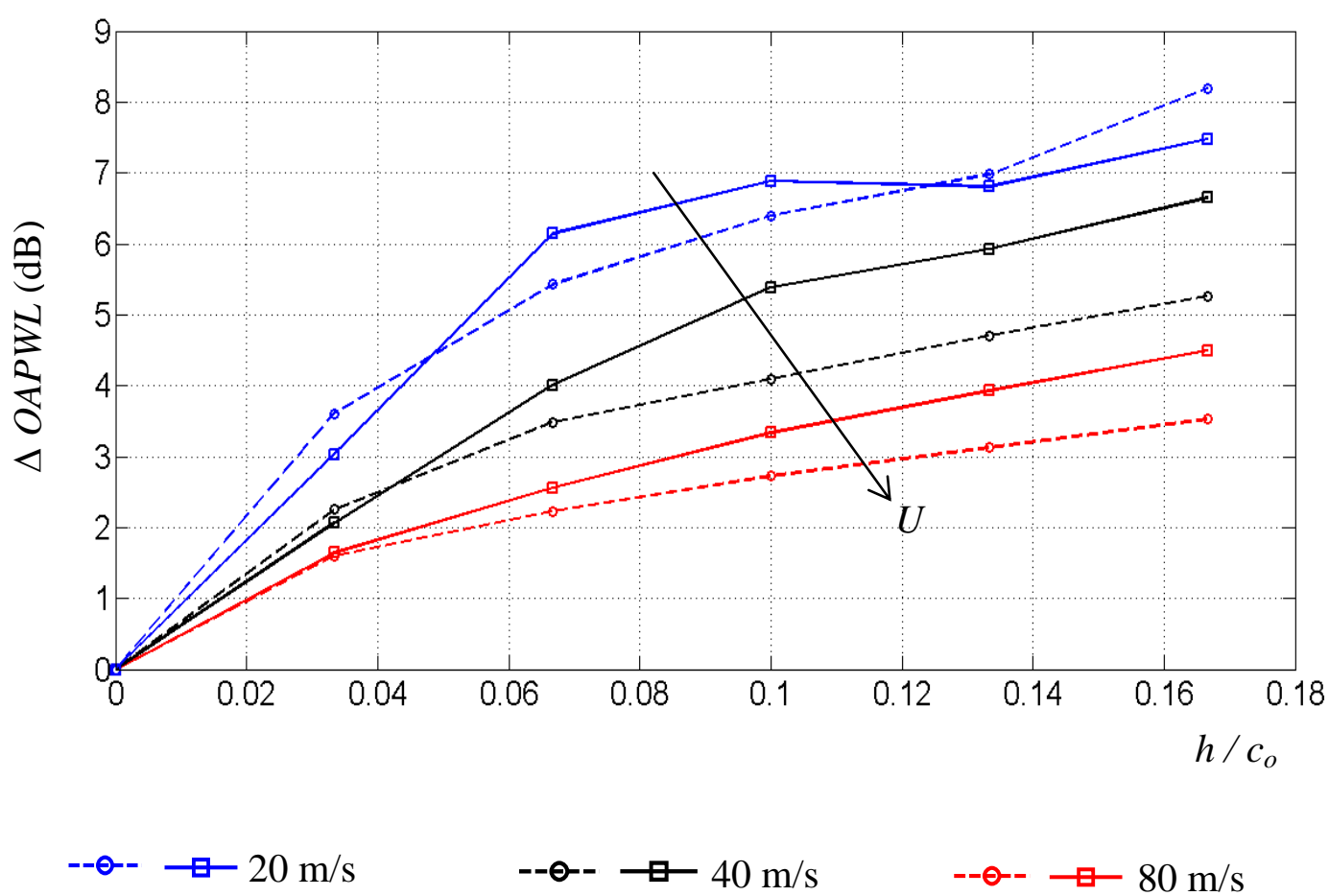

Fig. 9 Variation of $\triangle O A P W L$ with $h / c_{o}$ at $\lambda / c_{o}$ values of 0.033 (dashed lines) and 0.133 (solid lines) for various $U$ values 


\section{3 $\triangle P W L$ versus frequency and flow speed}

\subsubsection{Comparison of $\triangle P W L$ blow-down colour map of LE serrated flat plates}

In these studies, noise data is acquired as the fan speed is steadily reduced (or increased). Spectra are computed over short time intervals over which the change in flow speed is assumed to be negligible. In the present study, blow-down tests are conducted over a range of mean jet velocities between 55 to $30 \mathrm{~m} / \mathrm{s}$ for flat plates with and without $L E$-serrations.

The 'blow-down' maps of $\Delta P W L$ versus frequency and flow speed shown in Fig. 10 reveal the existence of a minimum frequency $f_{o}$ below which the noise reduction is negligible. This variation with flow speed appears to increase linearly with frequency and passes through the origin when extrapolated down to zero frequency. Such a variation suggests that significant noise reductions $(\geq 3 \mathrm{~dB}$ ) afforded by leading edge serrations are only possible when the serration amplitude $2 h$ is greater than a certain fraction, say $\alpha$, of a hydrodynamic wavelength $U / f_{o}$. Thus, the lower bound minimum frequency below which noise reductions are insignificant, may therefore be written as

$$
f_{o}=\frac{\alpha U}{2 h}
$$

where $\alpha$ is determined from the gradient of the dashed line in Fig. 10 as follows:

$$
\alpha=2 h \frac{\Delta f}{\Delta U}
$$

Values of $\alpha$ were obtained from blow-down maps for a range of $h$ and $\lambda$ values. No clear variation of $\alpha$ with $h$ and $\lambda$ was obtained. However, values of $\alpha$ were only found to vary in the narrow range of values between 0.4 and 0.6 for all the cases studied. There is some imprecision about the choice of straight line fit through the data and hence the value of $\alpha$, however. For all practical purposes, therefore, significant noise reductions are confined to the high frequencies, $f_{o}>U / 4 h$. 

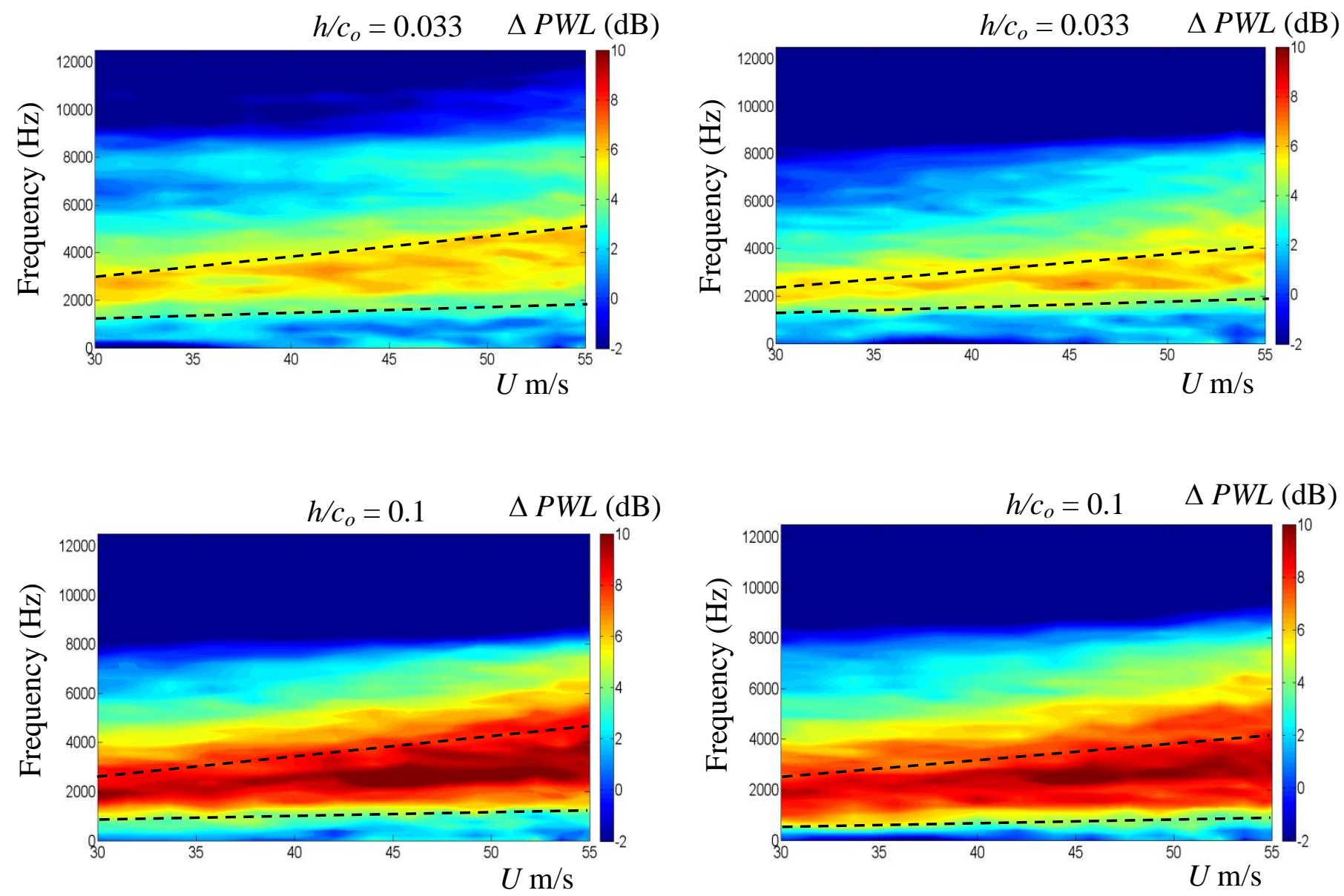

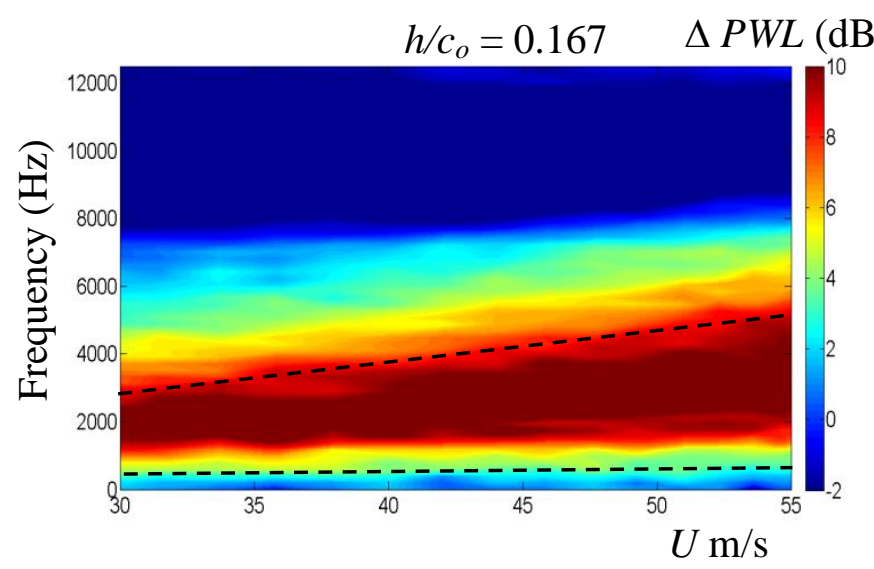

(a)

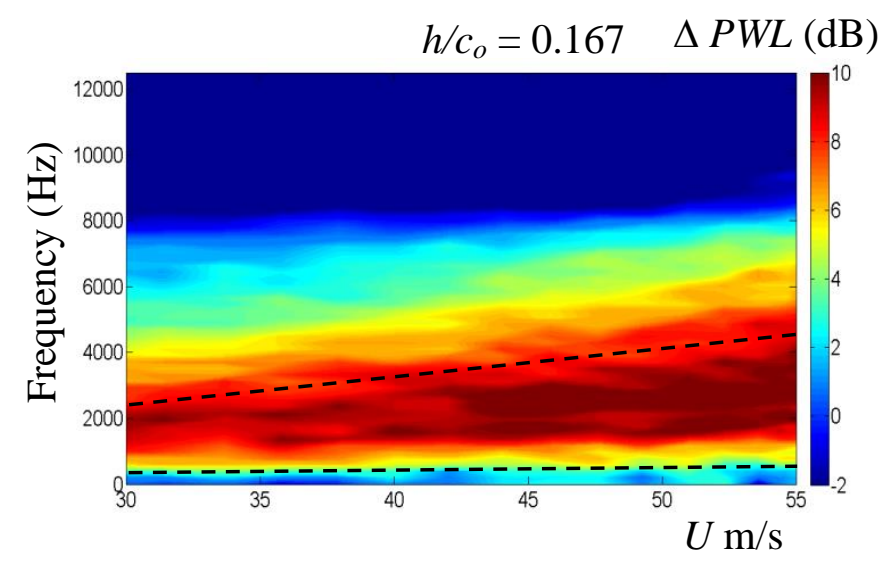

(b)

Fig. 10 Blow down map of $\Delta P W L$ variation for various amplitudes $\left(h / c_{o}\right)$ at wavelengths $\left(\lambda / c_{o}\right)$ of (a) 0.033 , (b) 0.1 
Figure 10 also shows the maximum frequency $f_{\max }$ (upper dashed line) where the noise reductions are maximum, above which we observe a drop in noise reductions. This drop is due to the dominance of trailing-edge self-noise over leading edge interaction noise. The $\triangle P W L$ blow-down map due to the flat plate serration is compared in Fig. 11 with that obtained from the NACA-65 aerofoil for identical serration parameters $h$ and $\lambda$. The same minimum frequency condition of Eq. (6) is observed for both flat plate and 3D aerofoil suggesting that the flat plate model is able to capture the essential physics of noise reductions achievable by the use of leading serrations.

Figure 11 (a) is a plot of the noise reductions versus frequency and flow speed due to the flat plate leading edge serration. It shows significant noise reductions (at least $3 \mathrm{~dB}$ ) between the lower-bound frequency $f_{o}$ defined by Eq. (6) and an upper frequency $f_{\max }$ of around $4 \mathrm{kHz}$ over the range of flow speeds, as indicated by the upper dashed line. Above this upper frequency value noise reductions decreases, which we now show is due to the dominance of trailing edge self-noise over leading edge interaction noise at those frequencies.

Measurements of the trailing edge self-noise were made by removing the turbulence grid from the nozzle and repeating the noise measurements versus frequency and flow speed, $P W L_{S N}(f, U)$. These measurements versus frequency and flow velocity were then subtracted from the total noise measurements $\left(P W L_{T O T}(f, U)\right)$ with the turbulence grid as follows:

$$
\Delta P W L_{(T O T-S N)}(f, U)=P W L_{T O T}(f, U)-P W L_{S N}(f, U)
$$

where $P W L_{T O T}(f, U)$ is the sum of interaction noise and self-noise.

This, difference $\Delta P W L_{(T O T-S N)}(f, U)$, given by Eq. (8), is due to the self-noise. The ' $0 \mathrm{~dB}$ ' contour of this difference represents the maximum frequency $f_{\max }$ at any given 
flow speed $U$ at which interaction noise and self-noise are equal, i.e., $\Delta P \operatorname{PL}_{(T O T-S N)}\left(f_{\max }, U\right) \sim$ 3. Thus, comparing Figs. 11 (a) and (b) for the flat plate, and Figs. 11 (c) and (d) for the aerofoil, indicates that the upper frequency limit where maximum noise reductions are achieved, are roughly the frequencies at which self-noise equals the interaction noise.

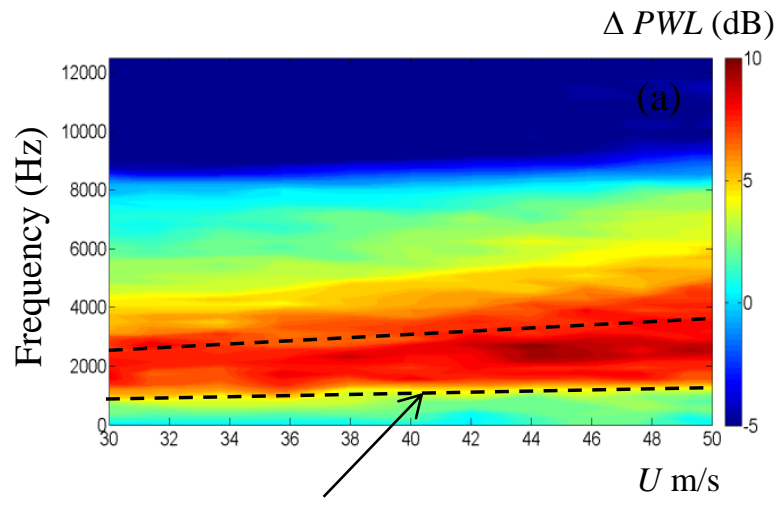

hf / $U=1 / 4$, Lower frequency band: Controlled by serration length/hydrodynamic wavelength

$\triangle P W L(\mathrm{~dB})$

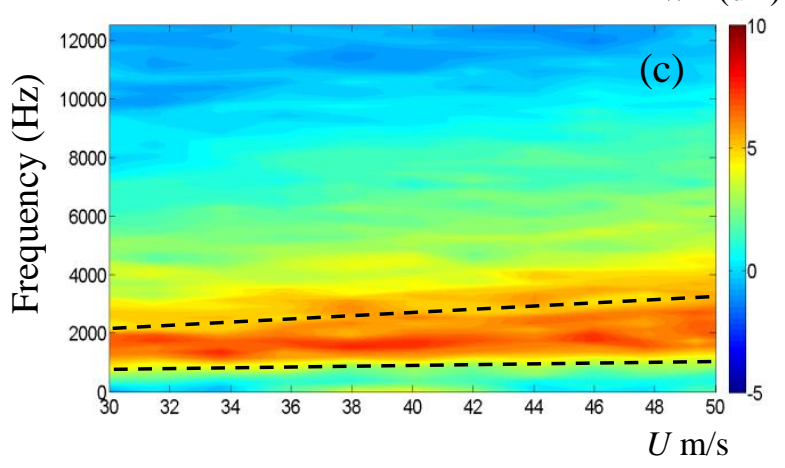

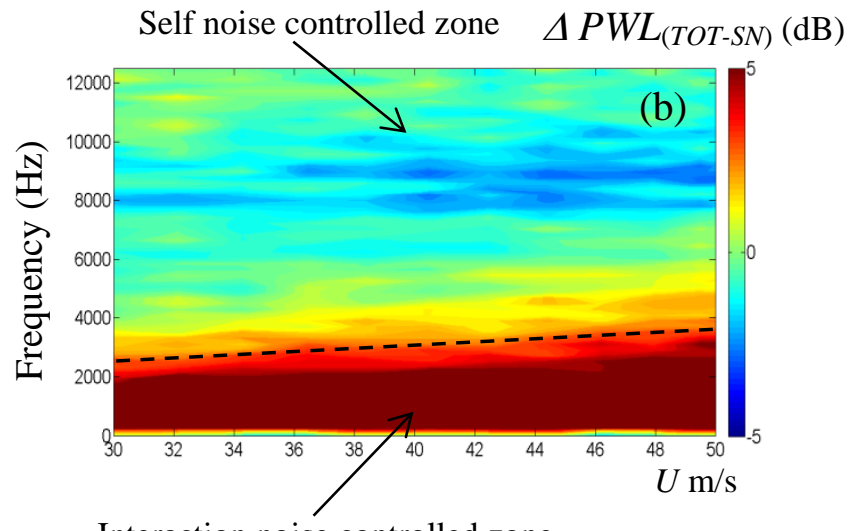

Interaction noise controlled zone

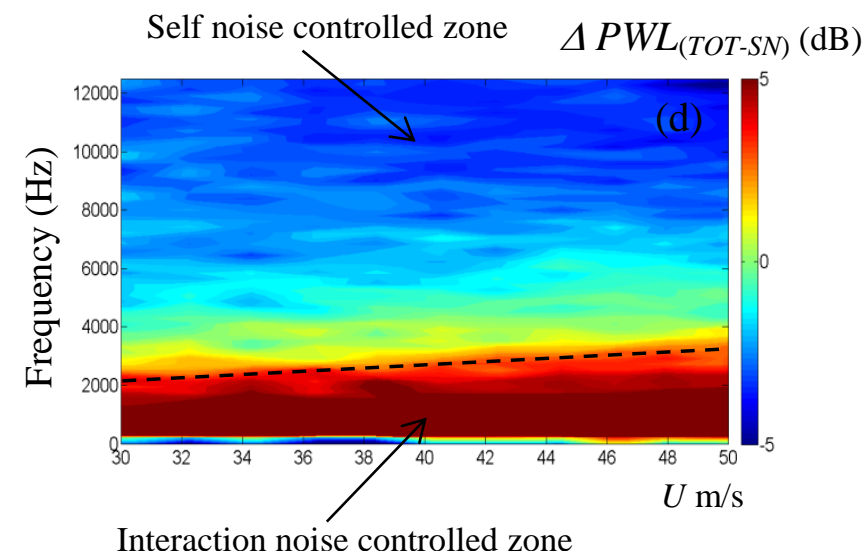

Fig. 11 Blow down maps of $\triangle P W L$ variation at $\lambda / c_{o}=0.067, h / c_{o}=0.067$ and the corresponding blow down maps of $\triangle P W L_{(T O T-S N)}\left(P W L_{T O T}(f, U)-P W L_{S N}(f, U)\right)$ for $(\mathrm{a}, \mathrm{b})$ Flat plate, (c, d) NACA-65 aerofoil 


\section{Numerical simulation of turbulent flow over baseline and $L E$ serrated flat plates}

\subsection{Computational method}

In this study numerical simulation are performed to further validate the experimental results. To this end, compressible Euler equations in general coordinates are solved using optimized fourth-order accurate pentadiagonal finite difference schemes [13]. In addition, $6^{\text {th }}$ order compact filters [14] are used for computational stability and parallelization is achieved using quasi-disjoint pentadiagonal matrices [15]. Communications are performed using MPI library in conjunction with a scalable algorithm suggested in [16]. Inflow and outflow conditions in horizontal and vertical directions and also non-penetrating wall condition for the plate surface are implemented using generalized characteristic boundary conditions (GCBC) [17] with periodic boundary conditions in span wise direction. A sponge-zone technique [5] is used to enforce the gust into the domain and also to damp any possible reflected disturbances from the GCBC back towards the physical domain. A 3D Inflow turbulence satisfying von Karman spectrum [18] is generated as suggested in [9] based on superposition of divergence-free vortices [19]. Far-field pressure is estimated using Ffowcs Williams and Hawkings $(F W-H)$ analogy written for the wind tunnel experiment (stationary source and observer in a mean flow) similar to formulation 1A of Farassat [20].

\subsection{Computational results}

The number of grid points used in the calculation is $N_{x}=1443, N_{y}=662$ and $N_{z}=33$ in each coordinate direction respectively. The computational grid for the baseline and sinusoidal $L E$ serrated flat plates are shown in Fig. 12. The domain extends from $-7 c^{*}$ to $11 c^{*}$ in $\mathrm{x}-$ direction, from $-7 c^{*}$ to $7 c^{*}$ in y-direction and $-L / 2 c^{*}$ to $L / 2 c^{*}$ in z-direction where $c^{*}=1$ is the non-dimensional chord and extends from $x / c_{o}=-0.5$ to 0.5 . A wavy leading edge case with $h / c_{o}=0.067, \lambda / c_{o}=0.133$ is considered. Only two wavelength of the plate are considered and a periodic boundary condition is implemented in the span-wise direction. The 
velocity fluctuations are generated using 300 artificial vortices on the domain boundary which are superimposed on the mean flow such that the resulting velocity field matches the von-Karman spectrum [9] up to $f c_{d} d a=5$. The vorticity magnitude $(|\omega|)$ contour near the leading edge of the flat plate airfoil shown in Fig. 13 clearly shows the worm-like structures of the homogenous and isotropic turbulence.

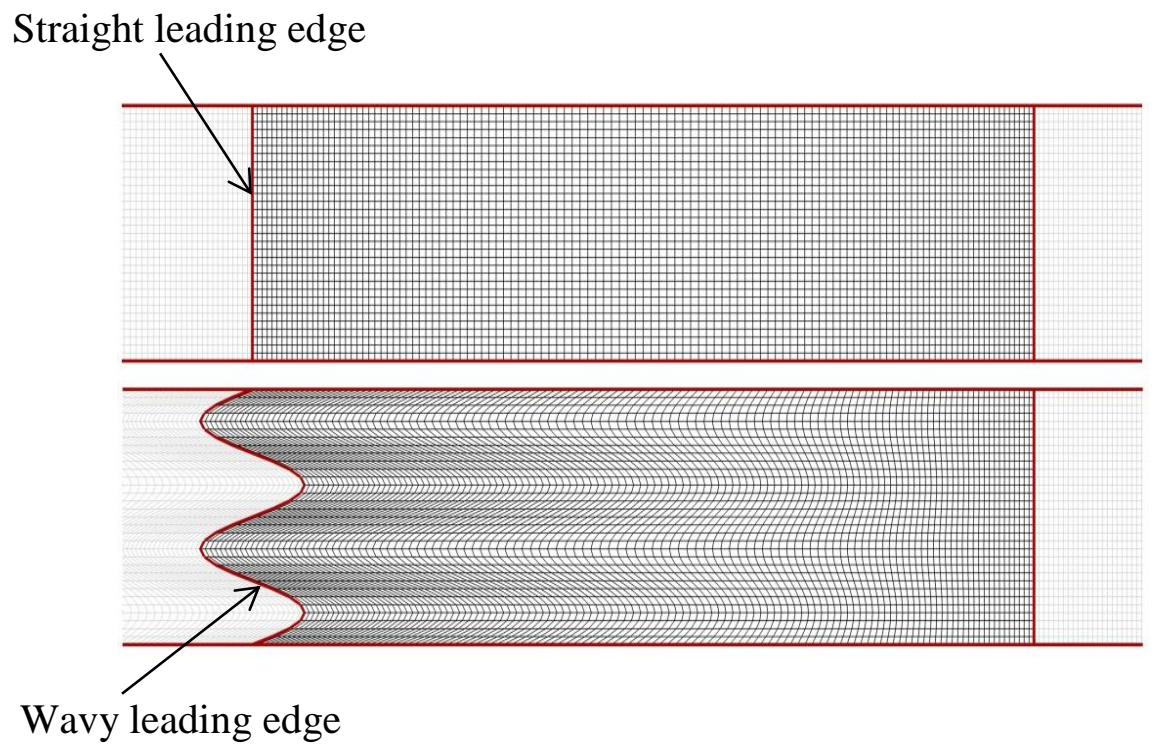

Fig. 12 Mesh generated for the straight and wavy leading edge flat plate cases

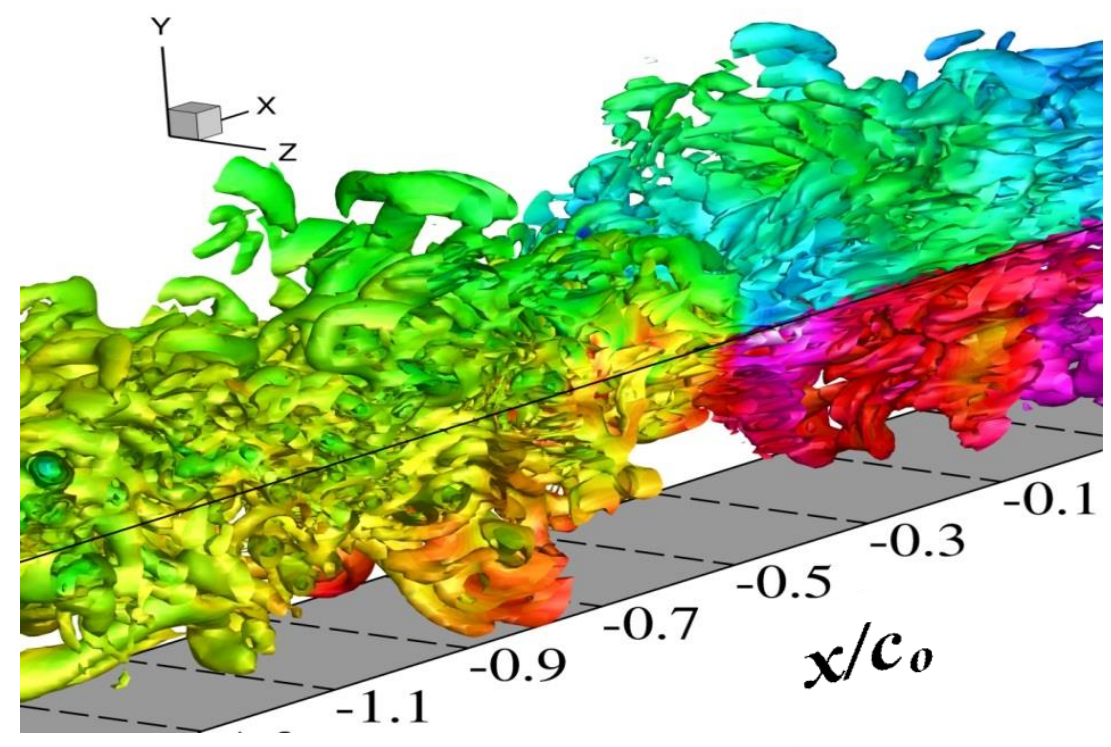

Fig. 13 Iso-surfaces of $|\omega| c / a$ between [0,6]. Iso-surfaces are plotted for 11 contour levels and are coloured by 11 levels of local pressure contours $\left(P^{\prime} / P_{\infty} \in\left[-2 \times 10^{-4}, 2 \times 10^{-4}\right]\right)$ 
The measured acoustic spectra for the baseline flat plate and the wavy leading edge cases $\left(h / c_{o}=0.067, \lambda c_{o}=0.133\right)$ are compared with those predicted using the surface pressure data obtained from the simulation in conjunction with the $F W-H$ equation in Fig. 14. Numerical spectrum is corrected to consider for the fact that the $F W-H$ equations are written for scattering of sound waves in a uniform mean flow whereas in the experiments, a shear layer exists and the acoustic pressure is recorded outside this shear layer in a quiescent medium. Therefore, to compare the $F W-H$ spectrum $(P S D)$ with the experimental measurements, it is multiplied by a factor of $\left(1-M^{2}\right)^{2}=0.89$ for the current simulations.

The acoustic spectra obtained from simulation (Fig. 14 (a)) shows reasonably good agreement with measurements up to around $1 \mathrm{kHz}$. In addition, as explained only two wavelengths of wavy leading edges are simulated. A periodic boundary condition is then used in both simulations and $F W-H$ calculations. The sound pressure reduction level $(\triangle S P L)$ obtained from computation is also compared with measured value in Fig. 14 (b). As mentioned earlier, large noise reductions which occur at peaks in the sharp edge (i.e., baseline) radiation spectra obtained from simulation at a non-dimensional frequency $f c_{\mathrm{o}} / a \sim 1.3$ matches well with the measured value of 1.2. The general trend of noise reduction levels obtained from prediction also shows reasonably good agreement with experiments as shown in Fig. 14 (b). Numerical predictions also confirm that the wavy leading edges are most effective for mid to high frequency range from about 2 to $10 \mathrm{kHz}$ although the experimental measurement show higher overall noise reduction levels. 


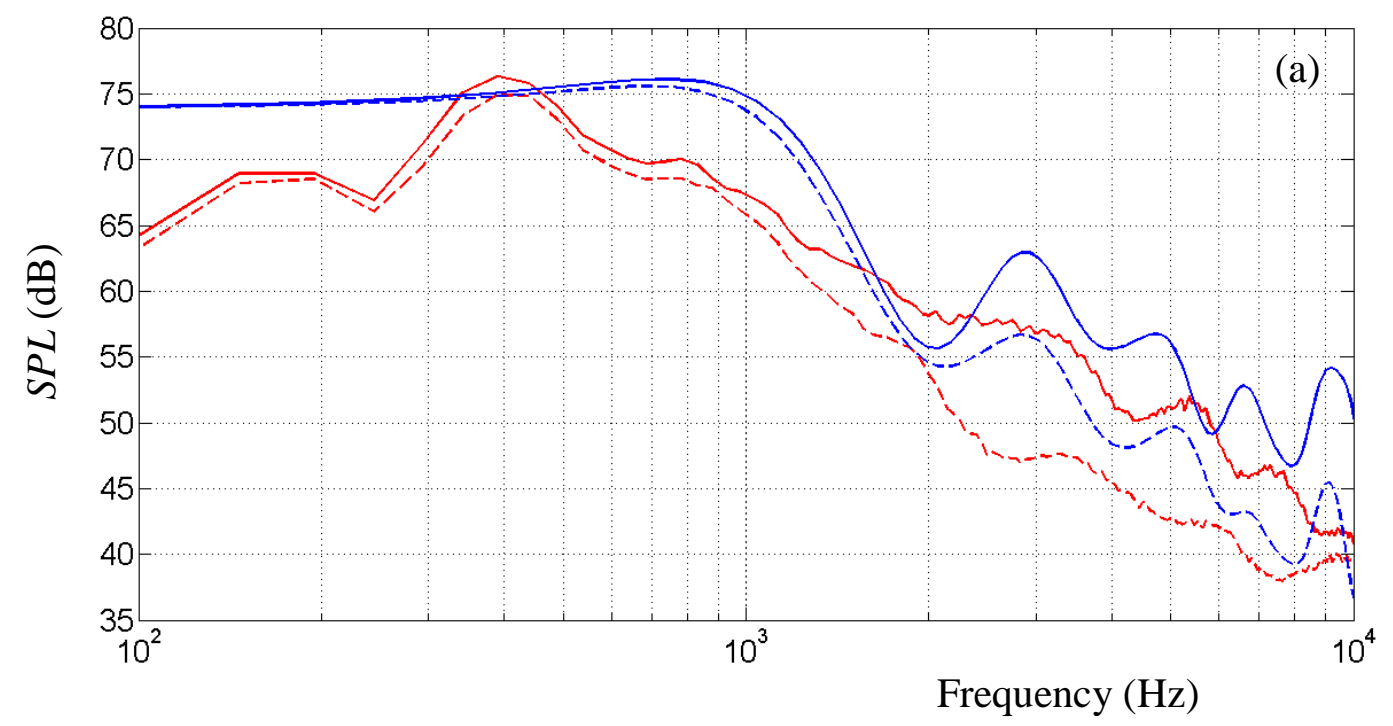

$\begin{array}{ll}\text { - Baseline experimental } & \text { Baseline computational } \\ \text {----- Serration experimental } & \text {----- Serration computational }\end{array}$

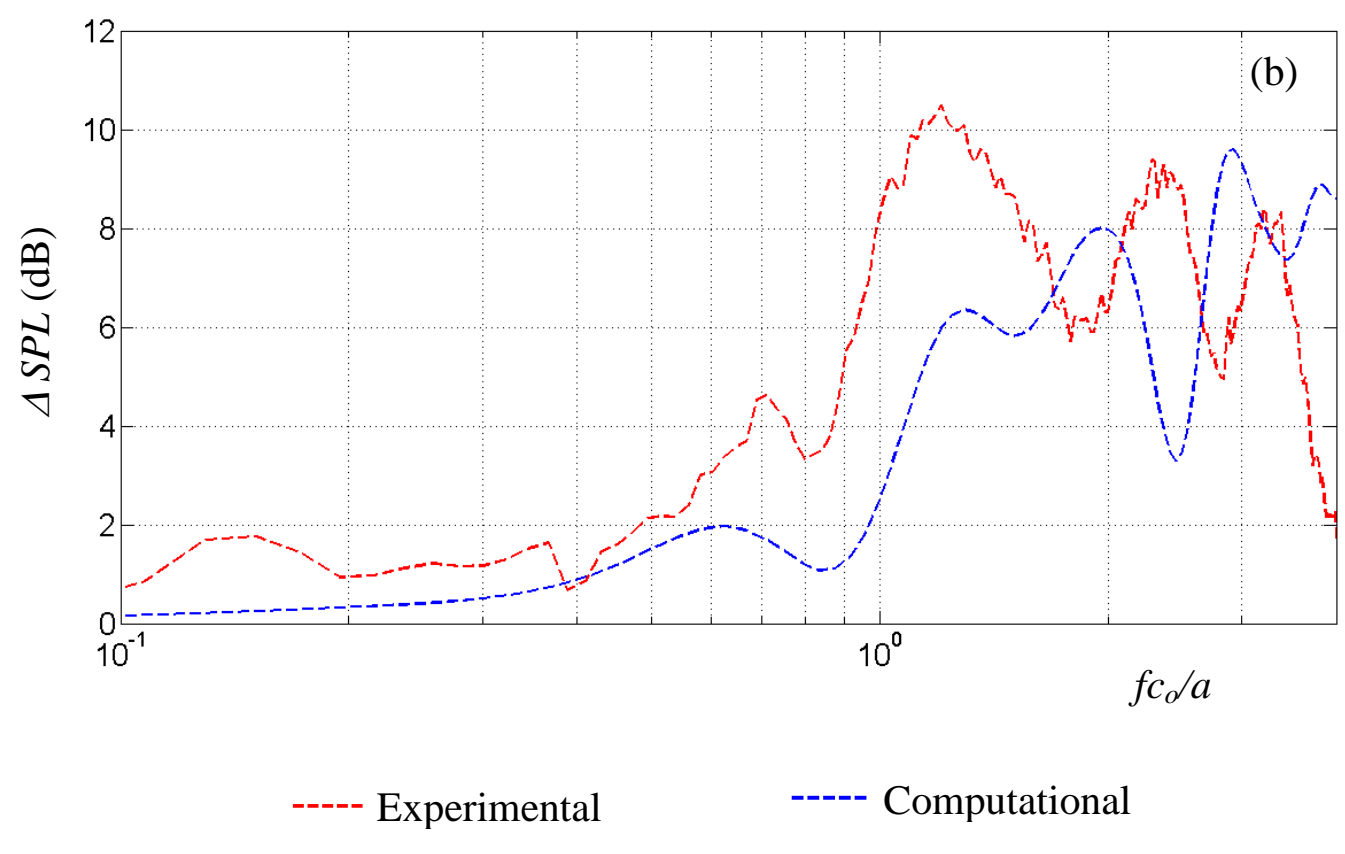

Fig. 14 Comparison of (a) sound pressure spectra and (b) reduction levels ( $\triangle S P L s)$, computed using the $F W$ - $H$ equations with experimental results at $\lambda / c_{o}=0.133, h / c_{o}=0.067$ 


\section{Conclusions}

The acoustic characteristics of flat plates with $L E$ serrations of different wavelengths and amplitudes are systematically studied and compared with un-serrated (i.e.; baseline) flat plate to understand and determine the effect of $L E$ serrations on the noise reduction characteristics. The acoustic characteristics of serrated flat plates are compared with un-serrated ones (i.e.; baseline flat plate) in terms of sound power reduction levels ( $\triangle P W L s)$.

The noise reduction performance of flat plates with sinusoidal leading edge serrations of different wavelengths and amplitudes has been investigated experimentally by locating them in a turbulent jet and measuring their far field noise. Their acoustic performance has been found to be similar to those achieved on realistic aerofoils with identical leading edge geometry. Flat plate experiments therefore provide a good model to the understanding of interaction noise on realistic aerofoils.

Comparison of the radiated sound power spectra with and without leading edge serrations have shown that significant noise reductions in leading edge noise can be achieved above a certain critical frequency. This lower frequency bound corresponds to the situation at which at least 0.5 of a hydrodynamic wavelength fits within the serration amplitude. This implies that the noise reduction mechanism is essentially due to interference of sound radiated along the edges.

The investigation has also demonstrated that the serration amplitude is the factor that mainly determines noise control performance. It is shown that overall noise reduction level in decibels varies logarithmically with the serration length. The reason for this dependence is not currently understood but it implies that improvements in noise control performance become progressively slower as the serration length is increased.

The paper has concluded with the results from some preliminary predictions of control performance obtained from an Euler solver. Predictions are within a few decibels of 
measurements and the noise reduction versus frequency exhibits the same oscillatory behaviour as the measurements.

\section{Acknowledgement}

The authors gratefully acknowledge that the current work (EP/J007633/1) has been supported by Engineering and Physical Sciences Research Council (EPSRC).

\section{References}

[1] Frank G. Collins, Boundary layer control on wings using sound and leading edge serrations, American Institute of aeronautics and Astronautics Journal, 19 (2) (1981) 129130.

[2] T. Bachmann, S. Klän, W. Baumgartner, M. Klaas, W. Schröder, and H. Wagner, Morphometric characterisation of wing feathers of the barn owl tyto alba pratincola and the pigeon columba livia. Frontiers in Zoology, 4(1):23 (2007).

[3] P.T. Soderman, Leading edge serrations which reduce the noise of low-speed rotors, NASA Technical Note, NASA TN D-7371, 1973.

[4] Alan S. Hersh, Paul T. Soderman, and Richard E. Hayden, Investigation of acoustic effects of leading edge serrations on airfoils, Journal of Aircraft, 11 (4) (1974) 197-202.

[5] Alex S.H. Lau, Sina Haeri, Jae Wook Kim, The effect of wavy leading edges on aerofoilgust interaction noise, Journal of Sound and Vibration, 332 (2013) 6234-6253.

[6] V. Clair, C. Polacsek, T. Le Garrec, G. Reboul, M. Gruber, and P. Joseph, Experimental and Numerical Investigation of Turbulence-Airfoil Noise Reduction Using Wavy Edges, American Institute of aeronautics and Astronautics Journal, 51 (11) (2013) 2695-2713 (doi: 10.2514/1.J052394).

[7] M. Roger, C. Schram, C. \& L. De Santana, Reduction of Airfoil Turbulence-Impingement Noise by Means of Leading-Edge Serrations and/or Porous Materials, $19^{\text {th }}$ AIAA/CEAS Aeroacoustics Conference, 27-29 May, Berlin, Germany, AIAA paper 2013-2108 (doi: 10.2514/6.2013-2108).

[8] R. K. Amiet, Acoustic radiation from an airfoil in a turbulent stream, Journal of Sound and Vibration, 41 (4) (1975) 407-420.

[9] S. Haeri, J. W. Kim, S. Narayanan, P. Joseph, 3D calculations of aerofoil-turbulence interaction noise and the effect of wavy leading edges, $20^{\text {th }}$ AIAA/CEAS Aeroacoustics Conference, 16-20 June 2014, Atlanta, GA, AIAA 2014-2325. 
[10] S. Narayanan , P. Joseph, S. Haeri, J. W. Kim, P Chaitanya, C. Polacsek, Noise reduction studies from the leading edge of serrated flat plates, $20^{\text {th }}$ AIAA/CEAS Aeroacoustics Conference, 16-20 June 2014, Atlanta, GA, AIAA 2014-2320.

[11] T. P. Chong, P.F. Joseph, and P. O. A. L. Davies, A parametric study of passive flow control for a short, high area ratio $90^{\circ}$ curved diffuser, Journal of Fluids Engineering, 130 (11) (2008), 111104-12.

[12] S. B. Pope, Turbulent flows, Cambridge university press, Fifth Edition (2008) 228-234.

[13] J. W. Kim, Optimised boundary compact finite difference schemes for computational aeroacoustics, Journal of Computational Physics 225 (2007) 995-1019.

[14] J. W. Kim, High-order compact filters with variable cut-off wavenumber and stable boundary treatment, Computers \& Fluids 39 (2010) 1168-82.

[15] J. W. Kim Quasi-disjoint pentadiagonal matrix systems for the parallelization of compact finite-difference schemes and filters, Journal of Computational Physics, 241 (2013) 168-194.

[16] S. Haeri and J.S. Shrimpton, CFDComm: An Optimized Library for Scalable Point-toPoint Communication for General CFD Applications, IEEE 14th International Conference on High Performance Computing and Communication (HPCC), (2012) 1001-1006, Liverpool, UK.

[17] J. W. Kim and D. J. Lee, Generalized Characteristic Boundary Conditions for Computational Aeroacoustics, AIAA Journal, 38 (2000) 2040-2049.

[18] A. Monin and A. Yaglom, Statistical Fluid Mechanics: Mechanics of Turbulence, vol. 2 MIT Press; 1975.

[19] A. Sescu and R. Hixon, Toward low-noise synthetic turbulence inflow conditions for aeroacoustic calculations. International Journal of Numerical Methods in Fluids, 73 (2013) 1001-1010.

[20] F. Farassat, Derivation of Formulation 1 and 1A of Farassat, Tech Rep, NASA TM2007-214853.

\section{List of Figures}

Fig. 1 (a) Flat plate model, (b) Schematic of smooth sinusoidal $L E$ serrations showing the parameters $h$ and $\lambda$, (c) A photograph of $L E$ serrated flat plate and jet nozzle inside the ISVR's anechoic chamber, (d) A photograph of the $L E$ serrated airfoil showing all the parameters (All dimensions are in $\mathrm{mm}$ ) 
Fig. 2 Photograph of jet nozzle and test setup inside the ISVR's anechoic chamber (All dimensions are in $\mathrm{mm}$ )

Fig. 3 (a) Turbulence grid located in the contraction section of the nozzle, (b) Comparison of the measured streamwise velocity spectrum $\left(\phi_{u и}\right)$ against Von Karman model for longitudinal isotropic turbulence, at $U=40 \mathrm{~m} / \mathrm{s}$

Fig. 4 Typical sound pressure spectra comparison of baseline and serrated-flat plates at $U=$ $60 \mathrm{~m} / \mathrm{s}$ for various amplitudes $\left(h / c_{o}\right)$ at a wavelength $\left(\lambda / c_{o}\right)$ of 0.067

Fig. 5 (a) Sound power level spectra comparison of baseline flat plate and serrated flat plate $\left(\lambda / c_{o}=0.067, h / c_{o}=0.067\right)$ with baseline NACA-65 aerofoil and serrated NACA-65 aerofoil $\left(\lambda / c_{o}=0.067, h / c_{o}=0.067\right)$ at $U=40 \mathrm{~m} / \mathrm{s}$, (b) Sound power reduction levels $(\triangle P W L)$ comparison of flat plate aerofoil and NACA- 65 aerofoil at $U=40 \mathrm{~m} / \mathrm{s}$

Fig. 6 Sound power reduction level $(\triangle P W L)$ comparison of (a) small amplitude serration $\left(\lambda / c_{o}=0.067, h / c_{o}=0.067\right)$, (b) large amplitude serration $\left(\lambda / c_{o}=0.067, h / c_{o}=0.167\right)$ for different jet velocities

Fig. 7 Variation of sound power reduction levels $(\triangle P W L)$ with non-dimensional frequency at $U=60 \mathrm{~m} / \mathrm{s}$ for various amplitudes $\left(h / c_{o}\right)$ at wavelengths $\left(\lambda / c_{o}\right)$ of (a) 0.033 , (b) 0.067 , (c) 0.1 , (d) 0.133

Fig. 8 Variation of $\triangle O A P W L$ with $h / c_{o}$ for various $U$ values of (a) $20 \mathrm{~m} / \mathrm{s}$, (b) $40 \mathrm{~m} / \mathrm{s}$, (c) 60 $\mathrm{m} / \mathrm{s},(\mathrm{d}) 80 \mathrm{~m} / \mathrm{s}$

Fig. 9 Variation of $\triangle O A P W L$ with $h / c_{o}$ at $\lambda / c_{o}$ values of 0.033 (dashed lines) and 0.133 (solid lines) for various $U$ values

Fig. 10 Blow down map of $\triangle P W L$ variation for various amplitudes $\left(h / c_{o}\right)$ at wavelengths $\left(\lambda / c_{o}\right)$ of (a) 0.033 , (b) 0.1

Fig. 11 Blow down maps of $\triangle P W L$ variation at $\lambda / c_{o}=0.067, h / c_{o}=0.067$ and the corresponding blow down maps of $\triangle P W L_{(T O T-S N)}\left(P W L_{T O T}(f, U)-P W L_{S N}(f, U)\right)$ for (a, b) Flat plate, (c, d) NACA-65 aerofoil

Fig. 12 Mesh generated for the straight and wavy leading edge flat plate cases

Fig. 13 Iso-surfaces of $|\omega| c / a$ between [0,6]. Iso-surfaces are plotted for 11 contour levels and are coloured by 11 levels of local pressure contours $\left(P^{\prime} / P_{\infty} \in\left[-2 \times 10^{-4}, 2 \times 10^{-4}\right]\right)$

Fig. 14 Comparison of (a) sound pressure spectra and (b) reduction levels ( $\triangle S P L s$ ), computed using the $F W$ - $H$ equations with experimental results at $\lambda / c_{o}=0.133, h / c_{o}=0.067$ 